\title{
Robust Metrics and Sensitivity Analyses for Meta-Analyses of Heterogeneous Effects
}

\author{
Maya B. Mathur ${ }^{1}$ and Tyler J. VanderWeele ${ }^{2}$ \\ ${ }^{1}$ Quantitative Sciences Unit, Stanford University, Palo Alto, CA, USA \\ ${ }^{2}$ Department of Epidemiology, Harvard T. H. Chan School of Public Health, \\ Boston, MA, USA
}

- Citation: Mathur, MB \& VanderWeele TJ (2020). Robust metrics and sensitivity analyses for meta-analyses of heterogeneous effects. Epidemiology, 31(3), 356-358. $[$ Link]

- Keywords: meta-analysis, effect sizes, heterogeneity, nonparametric, bootstrapping, confounding

- Conflicts of interest: The authors declare that they have no conflicts of interest.

- Reproducibility: All code and data required to reproduce the simulation study and applied example are publicly available (https://osf.io/6nyg8/). A general function to conduct the proposed analyses, prop_stronger, is publicly available in the $\mathrm{R}$ package MetaUtility.

- Source of funding: MBM and TVW were supported by NIH grant R01 CA222147. The funders had no role in the design, conduct, or reporting of this research. 


\begin{abstract}
We recently suggested new statistical metrics for routine reporting in random-effects meta-analyses to convey evidence strength for scientifically meaningful effects under effect heterogeneity. First, given a chosen threshold of meaningful effect size, we suggested reporting the estimated proportion of true effect sizes above this threshold. Second, we suggested reporting the proportion of effect sizes below a second, possibly symmetric, threshold in the opposite direction from the estimated mean. Our previous methods applied when the true effects are approximately normal, when the number of studies is relatively large, and when the proportion is between approximately 0.15 and 0.85 . Here, we additionally describe robust methods for point estimation and inference that perform well under considerably more general conditions, as we validate in an extensive simulation study. The methods are implemented in the $\mathrm{R}$ package MetaUtility (function prop_stronger). We describe application of the robust methods to conducting sensitivity analyses for unmeasured confounding in meta-analyses.
\end{abstract}

\title{
INTRODUCTION
}

We recently suggested new statistical metrics for routine reporting in random-effects metaanalyses to convey evidence strength for scientifically meaningful effects under effect heterogeneity $^{1}$. First, given a chosen threshold of meaningful effect size $(q)$, we suggested

reporting the estimated proportion of true effect sizes above this threshold $\left(\widehat{P}_{>q}\right)$. Second, we suggested reporting the proportion of effect sizes below a second, possibly symmetric, threshold in the opposite direction from the estimated mean. These metrics can help identify if: (1) there are few effects of scientifically meaningful size despite a "statistically significant" pooled point estimate; (2) there are some large effects despite an apparently null point estimate; or (3) strong effects in the direction opposite of the pooled estimate also regularly occur. Additionally, these metrics can sometimes adjudicate apparent "conflicts" between meta-analyses $^{2}$ and can convey evidence strength in multisite replication projects ${ }^{3}$.

We had proposed parametric estimation methods with asymptotic inference based on the delta method ${ }^{1}$; those methods applied when the true effects are approximately normal, when the number of studies is relatively large, and when $\widehat{P}_{>q}$ is between approximately 0.15 and 0.85 . Here, we additionally describe robust methods for point estimation and inference that perform well under more general conditions. 


\section{Methods}

Let $\theta_{i}, \widehat{\theta}_{i}$, and $\widehat{\sigma}_{i}$ respectively denote the true effect size, the point estimate, and the estimated standard error of the $i^{\text {th }}$ study. If the parameter $\theta_{i}$ for each study were known, then a simple nonparametric estimate $\widehat{P}_{>q}$ would simply be the sample proportion of $\theta_{i}$ greater than q. Given that the $\theta_{i}$ are in fact unknown, it would seem intuitive to instead compute the sample proportion of point estimates $\widehat{\theta}_{i}$ greater than $q$, but this approach is incorrect because dispersion in the $\widehat{\theta}_{i}$ reflects not only true effect heterogeneity, but also statistical error due to finite sample sizes in the meta-analyzed studies. Thus, the $\widehat{\theta}_{i}$ are overdispersed compared to the $\theta_{i}$ and would not themselves yield an unbiased estimate $\widehat{P}_{>q}$.

We therefore suggest computing the sample proportion using recently proposed "calibrated" estimates that have been appropriately shrunk to correct the overdispersion ${ }^{4}$. Let $\widehat{\mu}$ and $\widehat{\tau}^{2}$ represent classical Dersimonian-Laird ${ }^{5}$ meta-analytic estimates of the mean and variance of the true effects; these moment-based estimates do not require parametric assumptions. Wang et al. (2019) ${ }^{4}$ defined the calibrated point estimate for the $i^{\text {th }}$ study as:

$$
\widetilde{\theta}_{i}=\widehat{\mu}+\sqrt{\widehat{\tau}^{2} /\left(\widehat{\tau}^{2}+\widehat{\sigma}_{i}^{2}\right)}\left(\widehat{\theta}_{i}-\widehat{\mu}\right)
$$

and showed that $\operatorname{Var}\left(\widetilde{\theta}_{i}\right)=\widehat{\tau}^{2}$, as desired. Intuitively, the calibrated estimate $\widetilde{\theta}_{i}$ shrinks the point estimate $\widehat{\theta}_{i}$ toward the estimated meta-analytic mean $\widehat{\mu}$ with a degree of shrinkage that is inversely proportional to the study's precision: relatively imprecise estimates $\widehat{\theta}_{i}$ (i.e., those with large $\widehat{\sigma}_{i}$ ) receive strong shrinkage toward $\widehat{\mu}$, while relatively precise estimates receive less shrinkage and remain closer to their original values. Wang et al. (2019) ${ }^{4}$ demonstrated that the calibrated estimates can be used to construct approximately unbiased prediction intervals for small meta-analyses and for non-normal true effect distributions. For our purposes, we propose estimating the proportion of scientifically meaningful effect sizes as the sample proportion of calibrated estimates above $q$, i.e., $\widehat{P}_{>q}=\widehat{P}\left(\widetilde{\theta}_{i}>q\right)$. For inference, one can bootstrap pairs of $\left(\widehat{\theta}_{i}, \widehat{\sigma}_{i}\right)$ by drawing with replacement from the original sample 
and estimating in turn $\widehat{\mu}$ and $\widehat{\tau}^{2}, \widetilde{\theta}_{i}$ for each study, and finally $\widehat{P}_{>q}$. A bias-corrected and accelerated $(\mathrm{BCa})$ confidence interval ${ }^{6 ; 7}$ can then be constructed from the bootstrapped values of $\widehat{P}_{>q}$. (Naturally, analogous methods can be used to estimate the proportion of effects below another threshold.)

We also considered a simulation-based nonparametric method (here termed the "sign test method") that was originally designed to estimate a given percentile of interest (e.g., the median) of a distribution of effect sizes and to construct a confidence interval ${ }^{8}$. The method involves first conducting nonparametric hypothesis tests that are similar to sign tests for each of many possible values for the percentile of interest, then inverting the rejection region to form a confidence interval. This method can be straightforwardly repurposed to provide an estimate and confidence interval for the proportion of effects above a threshold, $P_{>q}$, as we show in the eAppendix.

We assessed all methods' performance in an extensive simulation study of 480 scenarios with a range of true effect distributions (including normal, highly skewed, bimodal, and heavytailed distributions), meta-analyses with 5 to 50 studies of varying size, varying heterogeneity, and true proportions from 0.05 to 0.50 . We included both realistic and extreme distributions of effect sizes in order to establish the boundary conditions under which the statistical methods would perform well. Some of the more extreme distributions may be unlikely to occur in practice, and conducting an aggregate meta-analysis when effects are clearly multimodal may be scientifically ill-advised in the first place. Details on the simulation study design appear in the eAppendix.

Based on the simulation results (eAppendix), we recommend reporting $\widehat{P}_{>q}$ and inference only for meta-analyses with at least 10 studies. In such meta-analyses, we recommend by default estimating $\widehat{P}_{>q}$ using the calibrated estimates; this method was the least biased for all distributions, though its root mean square error (RMSE) was sometimes higher than that 
of other methods. For inference, even when the effects appear normal, we recommend by default constructing the confidence interval by applying the bias-corrected and accelerated bootstrap to the calibrated estimates ("BCa-calibrated"); this method achieved nominal coverage in almost all scenarios and always achieved at least $90 \%$ coverage. The sign test method sometimes performed poorly when heterogeneity was low to moderate, and it offered few advantages over the BCa-calibrated method. We therefore do not recommend its use in practice to estimate or conduct inference for $P_{>q}$.

The BCa-calibrated method did sometimes lose considerable precision compared to the parametric method in certain scenarios in which the latter achieved approximately nominal coverage (e.g., see eFigure 7), so for large meta-analyses with apparently normal effects and estimating a proportion close to 0.50 , one might reasonably choose to substitute the parametric interval for the default BCa-calibrated interval. For example, in relatively small meta-analyses, estimating the amount of heterogeneity can be inherently imprecise ${ }^{9}$. This uncertainty propagates to the confidence interval for $P_{>q}$ and, in small meta-analyses, may result in confidence intervals that span most or all of the possible range [0,1]. Reporting confidence intervals in these settings may nevertheless be informative: a very wide confidence interval may instill appropriate circumspection about what can be learned regarding the distribution of true effects in a small meta-analysis, even if $\widehat{\mu}$ itself may have a narrow confidence interval (for example, see the applied example in the eAppendix). A wide confidence interval may further suggest the value of performing a larger meta-analysis when more literature becomes available. As an additional limitation, the BCa-calibrated interval may sometimes fail to converge for small meta-analyses. (When the BCa-calibrated interval fails to converge, it may seem attractive to construct a simpler bootstrapped confidence interval using percentiles of the bootstrapped calibrated estimates. However, we recommend against this method; additional simulation results suggested that it performed quite poorly.) The recommended methods are implemented in the function prop_stronger in the R package MetaUtility as of version 2.0.0. We illustrate this software and approach with an 
applied example in the eAppendix, where we also discuss extensions to sensitivity analysis for unmeasured confounding.

[1] Maya B Mathur and Tyler J VanderWeele. New metrics for meta-analyses of heterogeneous effects. Statistics in Medicine, 2018.

[2] Maya B Mathur and Tyler J VanderWeele. Finding common ground in meta-analysis "wars" on violent video games. Perspectives on Psychological Science, page 1745691619850104, 2019.

[3] Maya B Mathur and Tyler J VanderWeele. New statistical metrics for multisite replication projects. Under review. Preprint retrieved from https://osf.io/w89s5/.

[4] Chia-Chun Wang and Wen-Chung Lee. A simple method to estimate prediction intervals and predictive distributions: Summarizing meta-analyses beyond means and confidence intervals. Research Synthesis Methods, 10(2):255-266, 2019.

[5] Rebecca DerSimonian and Nan Laird. Meta-analysis in clinical trials. Controlled Clinical Trials, 7(3):177-188, 1986.

[6] Bradley Efron. Better bootstrap confidence intervals. Journal of the American Statistical Association, 82(397):171-185, 1987.

[7] James Carpenter and John Bithell. Bootstrap confidence intervals: When, which, what? A practical guide for medical statisticians. Statistics in Medicine, 19(9):1141-1164, 2000.

[8] Rui Wang, Lu Tian, Tianxi Cai, and LJ Wei. Nonparametric inference procedure for percentiles of the random effects distribution in meta-analysis. The Annals of Applied Statistics, 4(1):520, 2010.

[9] Areti Angeliki Veroniki, Dan Jackson, Wolfgang Viechtbauer, Ralf Bender, Jack Bowden, Guido Knapp, Oliver Kuss, Julian Higgins, Dean Langan, and Georgia Salanti. Methods to estimate the between-study variance and its uncertainty in meta-analysis. Research Synthesis Methods, 2015. 


\title{
eAppendix: Robust Metrics and Sensitivity Analyses for Meta-Analyses of Heterogeneous Effects
}

\author{
Contents
}

\begin{tabular}{|lll}
\hline 1 & Theory & 3
\end{tabular}

1.1 Sign test method . . . . . . . . . . . . . . . . . . . . 3

1.2 Sensitivity analysis for unmeasured confounding . . . . . . . . . . . . . . . 4

1.2 .1 Setting and notation . . . . . . . . . . . . . . . 4

1.2.2 Proportion of studies with scientifically meaningful effect sizes as a function of the bias factor . . . . . . . . . . . . . . . 5

1.2.3 Bias factor or confounding strength required to reduce proportion of scientifically meaningful effect sizes to below a threshold . . . . . . . 6

1.2.4 Meta-analyses including both randomized and observational studies . 7

\begin{tabular}{lll}
\hline 2 & Simulation study & 7
\end{tabular}

2.1 Methods . . . . . . . . . . . . . . . . . . . . . . . . . 7

2.2 Summary of results . . . . . . . . . . . . . . . . . . . . . . . . 9

2.3 All coverage results by distribution . . . . . . . . . . . . . . . 12

2.4 All confidence interval width results by distribution . . . . . . . . . . . . 16

2.5 All RMSE results by distribution . . . . . . . . . . . . . . . . . . . . . . . . 20

2.6 All bias results by distribution . . . . . . . . . . . . . . . . . . 24 
\begin{tabular}{llr}
\hline 3 & Applied example & 28
\end{tabular}

$\begin{array}{lrl}4 \text { Software } & 31\end{array}$ 


\section{THEORY}

\subsection{Sign test method}

In addition to the calibrated estimation method described in the main text, we considered an additional robust method that follows straightforwardly from repurposing existing methods for conducting inference on the percentiles of a heterogeneous effect distribution ${ }^{1}$. Let $k$ denote the number of studies in the meta-analysis and let $\widehat{B}_{i}=1\left\{\widehat{\theta}_{i}<q\right\}-1\left\{\widehat{\theta}_{i}>q\right\}$. Wang et al. (2010) proposed the test statistic ${ }^{\mathbb{1}}$ :

$$
\widehat{T}(q)=\sum_{i=1}^{k}\left|\Phi\left(\left(q-\widehat{\theta}_{i}\right) / \widehat{\sigma}_{i}\right)-1 / 2\right| \widehat{B}_{i}=\sum_{i=1}^{k}\left\{\Phi\left(\left(q-\widehat{\theta}_{i}\right) / \widehat{\sigma}_{i}\right)-1 / 2\right\}
$$

where $\Phi$ denotes the cumulative distribution function of the standard normal distribution. To provide intuition, the term $\Phi\left(\left(q-\widehat{\theta}_{i}\right) / \widehat{\sigma}_{i}\right)$ represents the asymptotic coverage level of the confidence interval $[-\infty, q]$ for $\theta_{i}$, that is, $P\left(q>\theta_{i}\right) !^{a}$ Thus, the term in the absolute value serves as a precision weight for the contribution $\widehat{B}_{i}$ in that it compares the precision of study $i$ (specifically with respect to the threshold $q$ ) to that of a study that is maximally uninformative in the sense that $P\left(q>\theta_{i}\right)=1 / 2$. To test a null hypothesis equivalent to $H_{0}: 1-P_{>q}=p^{*}$ for a fixed percentile $p^{*}$, Wang et al. $(2010)^{\mathbb{1}}$ simulated a reference distribution for $\widehat{T}(q)$ under $H_{0}$, calling the reference test statistic $\widehat{T}^{*}(q)$ :

$$
\widehat{T}^{*}(q)=\sum_{i=1}^{k}\left|\Phi\left(\left(q-\widehat{\theta}_{i}\right) / \widehat{\sigma}_{i}\right)-1 / 2\right| \Delta_{i}
$$

where $\Delta_{i}$ is a null counterpart to $\widehat{B}_{i}$ that is simulated to equal 1 with probability $p^{*}$ and to equal -1 with probability $1-p^{*}$. Wang et al. $(2010)^{[2]}$ showed that this simulated distribution approximates the true distribution under $H_{0}$ when the sample size in the meta-analyzed studies is large, though without requiring asymptotics on the number of meta-analyzed studies $(k)$. These results designed for conducting inference on a fixed percentile of interest also allow straightforward inference and point estimation for the proportion of effects above a threshold, $P_{>q}$. To do so, one can specify a grid of $M$ values $\left(p_{1}^{*}, \cdots, p_{M}^{*}\right)$ ranging from 0

\footnotetext{
${ }^{a}$ Specifically, let $n_{i}$ be the sample size in the $i^{t h}$ study. Then $\widehat{\theta}_{i} \underset{n_{i} \rightarrow \infty}{\stackrel{D}{\longrightarrow}} N\left(\theta_{i}, \sigma_{i}^{2}\right)$. Consider the coverage of a confidence interval for $\widehat{\theta}_{i}$ with lower bound $\widehat{\theta}_{i}-c \widehat{\sigma}_{i}$ for an arbitrary constant $c>0$. Asymptotically, the probability that the lower bound is too high to cover $\theta_{i}$ is $P\left(\widehat{\theta}_{i}-c \widehat{\sigma}_{i}>\theta_{i}\right) \underset{n_{i} \rightarrow \infty}{\stackrel{p}{\longrightarrow}} \Phi(-c)$. Setting $q=\widehat{\theta}_{i}-c \widehat{\sigma}_{i}$ yields $P\left(q>\theta_{i}\right)=\Phi\left(\left(q-\widehat{\theta}_{i}\right) / \widehat{\sigma}_{i}\right)$, as desired.
} 
to 1 and conduct a level- $\alpha$ test of each hypothesis $H_{0, m}: 1-P_{>q}=p_{m}^{*}$ by simulating many iterates of the reference statistics $\widehat{T}^{*}(q)$, whose distribution depends on $p^{*}$ via the random binary variable $\Delta_{i}$. The set of $1-p_{m}^{*}$ that are not rejected at level $\alpha$ form the $100 \times(1-\alpha) \%$

confidence interval for $\widehat{P}_{>q}$, and a point estimate $\widehat{P}_{>q}$ can be defined as the value $1-p_{m}^{*}$ with the largest $p$-value (which we term the "sign test max" in the simulation study).

\subsection{Sensitivity analysis for unmeasured confounding}

We previously developed sensitivity analyses for unmeasured confounding in meta-analyses; these methods quantified the proportion of effects of scientifically meaningful magnitude, $\widehat{P}_{>q}$, under a specified amount of unmeasured confounding ${ }^{3}$. We also developed converse methods to estimate the strength of confounding capable of reducing $\widehat{P}_{>q}$ itself to below a chosen threshold ${ }^{3}$. These methods used parametric point estimation and inference that generalized the parametric methods described in the main text here ${ }^{4}$. These sensitivity analysis methods can be conducted robustly using the present calibration-based methods as follows.

\subsubsection{Setting and notation}

This background material is partly reproduced from our previous work regarding sensitivity analysis using parametric methods, where we provide more detail, intuition, and guidance on practical interpretation 53 . Let $X$ denote a binary exposure, $Y$ a binary outcome, $Z$ a vector of measured confounders, and $U$ one or more unmeasured confounders ${ }^{5}$. Consider the point estimate for a single meta-analyzed study on the relative risk scale; other effect size measures, such as standardized mean differences and odds ratios, can be approximately converted to relative risks to allow application of these methods, as when conducting the sensitivity analyses parametrically ${ }^{\underline{3}}$. Let:

$$
R R_{X Y \mid z}=\frac{P(Y=1 \mid X=1, Z=z)}{P(Y=1 \mid X=0, Z=z)}
$$

be the confounded relative risk $(R R)$ of $Y$ for $X=1$ versus $X=0$ conditional or stratified on the measured confounders $Z=z$. Let its unconfounded counterpart standardized to the population be:

$$
R R_{X Y \mid z}^{\prime}=\frac{\sum_{u} P(Y=1 \mid X=1, Z=z, U=u) P(U=u \mid Z=z)}{\sum_{u} P(Y=1 \mid X=0, Z=z, U=u) P(U=u \mid Z=z)}
$$


Define the ratio of the confounded to the unconfounded relative risks as $B=R R_{X Y \mid z} / R R_{X Y \mid z}^{\prime}$; this "bias factor" can be sharply bounded as follows". Let:

$$
R R_{X u}=P(U=u \mid X=1) / P(U=u \mid X=0)
$$

Define the first sensitivity parameter as $R R_{X U}=\max _{u}\left(R R_{X u}\right)$; that is, the maximal relative risk of $U=u$ for $X=1$ versus $X=0$ across strata of $U$. (If $U$ is binary, this is just the relative risk relating $X$ and $U$.) Next, for each stratum $x$ of $X$, define a relative risk of $U$ on $Y$, maximized across all possible contrasts of $U$ :

$$
R R_{U Y \mid X=x}=\frac{\max _{u} P(Y=1 \mid X=x, U=u)}{\min _{u} P(Y=1 \mid X=x, U=u)}, x \in\{0,1\}
$$

Define the second sensitivity parameter as $R R_{U Y}=\max \left(R R_{U Y \mid X=0}, R R_{U Y \mid X=1}\right)$. That is, considering both strata of $X$, it is the largest of the maximal relative risks of $U$ on $Y$ conditional on $X$. Others ${ }^{\frac{5}{5}}$ showed that when $B \geq 1$, then $B$ itself is bounded above by:

$$
B \leq \frac{R R_{X U} \cdot R R_{U Y}}{R R_{X U}+R R_{U Y}-1}
$$

and that when $B \leq 1$, the same bound holds for $1 / B$. Thus, defining the "worst-case" bias factor as $B^{+}=\frac{R R_{X U} \cdot R R_{U Y}}{R R_{X U}+R R_{U Y}-1}$, a sharp bound for the unconfounded effect when the observed $R R_{X Y \mid z} \geq 1$ is $R R_{X Y \mid z}^{\prime} \geq R R_{X Y \mid z} / B^{+}$, and a sharp bound when $R R_{X Y \mid z} \leq 1$ is $R R_{X Y \mid z}^{\prime} \leq R R_{X Y \mid z} \times B^{+}$.

\subsubsection{Proportion of studies with scientifically meaningful effect sizes as a func- tion of the bias factor}

Here, we consider the case in which $B$ is assumed to be homogeneous across studies; that is, all studies are subject to the same degree of unmeasured confounding, albeit possibly due to different unmeasured confounders. Assuming homogeneous, rather than heterogeneous, bias across studies yields conservative sensitivity analyses in some settings; see Mathur \& VanderWeele's (2019) ${ }^{3}$ Table 1 for details.

As one metric of sensitivity to unmeasured confounding, we can estimate the proportion of unconfounded effects stronger than $q$ when all studies have bias factor $B$ (i.e., all studies' relative risk estimates are shifted away from the null by a factor of $B$ due to unmeasured confounding). This quantity, here denoted $\widehat{P}_{>q}(B)$, can be robustly estimated using the 
calibrated estimates as follows. First, for a chosen bias factor $B$, define a bias-corrected point estimate for each study on the log relative risk scale as $\widehat{\theta}_{i}^{\prime}=\log \left(R R_{X Y \mid z} / B\right)=\widehat{\theta}_{i}-\log B$ if $\widehat{\mu}>0$ (i.e., the confounded pooled point estimate on the log relative risk scale is apparently causative) or as $\widehat{\theta}_{i}^{\prime}=\log \left(R R_{X Y \mid z} \times B\right)=\widehat{\theta}_{i}+\log B$ if $\widehat{\mu}<0$ (i.e., the confounded pooled point estimate is apparently preventive). Similarly, define the bias-corrected pooled point estimate $\widehat{\mu}^{\prime}=\widehat{\mu}-\log B$ if $\widehat{\mu}>0$ and as $\widehat{\mu}^{\prime}=\widehat{\mu}+\log B$ if $\widehat{\mu}<0$. Next, use the bias-corrected point estimates $\widehat{\theta}_{i}^{\prime}$ and pooled point estimate $\widehat{\mu}^{\prime}$ to calculate bias-corrected calibrated estimates on the $\log$ relative risk scale as $\widetilde{\theta}_{i}^{\prime}=\widehat{\mu}^{\prime}+\sqrt{\widehat{\tau}^{2} /\left(\widehat{\tau}^{2}+\widehat{\sigma}_{i}^{2}\right)}\left(\widehat{\theta}_{i}^{\prime}-\widehat{\mu}^{\prime}\right)$. (Note that because $B$ is constant across studies, no bias correction is needed for $\widehat{\tau}^{2}$.) Then $\widehat{P}_{>q}(B)$ can be straightforwardly estimated using the sample proportion of bias-corrected point estimates that are stronger than $q$, i.e., $\widehat{P}_{>q}(B)=\widehat{P}\left(\widetilde{\theta}_{i}^{\prime}>q\right)$. A confidence interval can be obtained via bias-corrected and accelerated $(\mathrm{BCa})$ bootstrapping as described in the main text, resampling the pairs $\left(\widehat{\theta}_{i}^{\prime}, \widehat{\sigma}_{i}\right)$.

\subsubsection{Bias factor or confounding strength required to reduce proportion of sci- entifically meaningful effect sizes to below a threshold}

As a second sensitivity analysis metric, we previously proposed reporting the minimum bias factor in each study that would be required to reduce to less than $r$ (e.g., 0.10) the proportion of effects of scientifically meaningful magnitude ${ }^{3}$. This metric, denoted $\widehat{T}(r, q)$, can be robustly estimated using a simple grid search across values of $B$. That is, for each of a grid of candidate values for the bias factor $B$, such as $(1,1.01,1.02, \ldots)$, apply the methods described in Section 1.2 .2 to estimate $\widehat{P}_{>q}(B)$. Then, $\widehat{T}(r, q)$ is simply the bias factor such that $\widehat{P}_{>q}(B)$ is exactly equal to the chosen proportion $r$; that is, $\widehat{T}(r, q)=B: \widehat{P}_{>q}(B)=r$.

Recall that $B^{+}$, the upper bound on $B$, is a function of two sensitivity parameters that characterize the strengths of association between the unmeasured confounder(s) and the exposure $\left(R R_{X U}\right)$ and between the unmeasured confounder(s) and the outcome $\left(R R_{U Y}\right)$. If these two sensitivity parameters are assumed to be equal to one another $\underline{\underline{63}}$, the sensitivity analysis metric $\widehat{T}(r, q)$ can alternatively be parameterized on the more intuitive confounding strength scale (i.e., the values of both $R R_{X U}$ and $R R_{U Y}$ ). Consider the minimum confounding strength required to reduce to less than $r$ the proportion of studies with scientifically meaningful effect sizes. This quantity, denoted $\widehat{G}(r, q)$, can be obtained as a simple transformation of $\widehat{T}(r, q)$ as follows: $\widehat{G}(r, q)=\widehat{T}(r, q)+\sqrt{(\widehat{T}(r, q))^{2}-\widehat{T}(r, q)}$. This metric is closely analogous to the "E-value" for an individual study 
Confidence intervals for $\widehat{T}(r, q)$ or $\widehat{G}(r, q)$ can be constructed via bootstrapping by resampling the bias-corrected point estimates, $\widehat{\theta}_{i}^{\prime}$, estimating the desired quantity $(\widehat{T}(r, q)$ or $\widehat{G}(r, q))$ for each resample, and constructing confidence intervals using the BCa method. In practice, the same set of resamples could be used for $\widehat{P}_{>q}(B), \widehat{T}(r, q)$, and $\widehat{G}(r, q)$.

\subsubsection{Meta-analyses including both randomized and observational studies}

The present robust methods also allow straightforward extension to meta-analyses in which some studies (e.g., randomized studies) are assumed to have no unmeasured confounding, while other studies are assumed to be subject to unmeasured confounding of strength $B$. For this setting, set the bias-corrected point estimates $\widehat{\theta}_{i}^{\prime}$ equal to their observed values $\left(\widehat{\theta}_{i}\right)$ for the randomized studies and to $\widehat{\theta}_{i}-\log B$ or $\widehat{\theta}_{i}+\log B$, as described above, for the observational studies. Then, meta-analyze these bias-corrected point estimates to arrive at a bias-corrected pooled point estimate and heterogeneity estimate, $\widehat{\mu}^{\prime}$ and $\widehat{\tau}^{2 \prime}$, and use these to compute the calibrated estimates. (Note that because $B$ is no longer constant across all studies, it is now necessary to estimate $\widehat{\tau}^{2 \prime}$ using the calibrated, bias-corrected estimates.) Estimation and inference for $\widehat{P}_{>q}(B), \widehat{T}(r, q)$, and $\widehat{G}(r, q)$ would then proceed as above.

\section{Simulation STUdy}

\subsection{Methods}

The simulation study assessed the performance of point estimation and inference methods for $\widehat{P}_{>q}$ without confounding. We fixed the mean of the true population effects to $\mu=0.50$ on the mean difference scale while varying the number of studies $(k)$ between 5 and 50 , the heterogeneity (the variance of true population effects) $\tau^{2} \in\{0.01,0.04,0.25\}$, and the distribution of total sample sizes within each study (either $N \sim \operatorname{Unif}(100,200)$ or $N \sim \operatorname{Unif}(800,900))$. For each of $k$ meta-analyzed studies, we generated a true effect, $\theta_{i}$, on the raw mean difference scale from a normal distribution, a scaled and shifted $t$-distribution with 3 degrees of freedom, a bimodal uniform mixture distribution, or a shifted exponential distribution. For all distributions, we chose the parameters to provide the desired mean of $\mu=0.50$ and heterogeneity $\tau^{2}$. Figure 1 shows example data depicting true population effects simulated from each of the four distributions for each value of $\tau^{2}$. 
We then simulated subject-level data for a control group with mean 0 and for a treatment with mean $\theta_{i}$; each group was of size $N / 2$ with a standard deviation of 1 . Thus, the within-study standard error of the estimated mean difference, $\widehat{\theta}_{i}$, was approximately $\widehat{\sigma}_{i}=\sqrt{4 / N}$. For the meta-analysis, the proportion of the total variance attributable to effect heterogeneity (termed $\left.I^{2}\right)$ was approximately $\tau^{2} /\left(\tau^{2}+4 / E[N]\right)$. We chose values of $q$ to result in true proportions $P_{>q}$ of $0.05,0.10,0.20$, and 0.50 .

We ran scenarios representing all 480 possible combinations of the varying parameters, using 5,000 iterates to estimate both types of bootstrap confidence interval and 2,000 iterates to estimate the reference distribution for the sign test method ${ }^{11}$. We ran at least 500 simulation iterates per scenario. For inference, we assessed the coverage and width of $95 \%$ confidence intervals constructed by computing $\widehat{P}_{>q}$ from calibrated estimates in bootstrapped datasets as described above ("BCa-calibrated"), constructed using the delta method ("Parametric") ${ }^{\mathbb{4}}$, and constructed by estimating $\widehat{P}_{>q}$ parametrically in bootstrapped datasets ("BCa-parametric") as we previously described ${ }^{4}$. For point estimation, we assessed the root mean squared error (RMSE) and absolute bias of three methods: the parametric method ("Parametric"), the sample proportion based on the calibrated estimates ("Calibrated"), and the value maximizing the $p$-value of the sign test as described above ("Sign test max.").

\begin{tabular}{lccc}
\hline & $\tau^{2}=0.01$ & $\tau^{2}=0.04$ & $\tau^{2}=0.25$ \\
\hline$E[N]=150$ & 0.27 & 0.60 & 0.90 \\
$E[N]=850$ & 0.68 & 0.89 & 0.98 \\
\hline
\end{tabular}

eTable 1: Approximate values of relative heterogeneity $\left(I^{2}\right)$ for each combination of simulation parameters regarding the mean within-study sample size $(E[N])$ and heterogeneity $\left(\tau^{2}\right)$. 

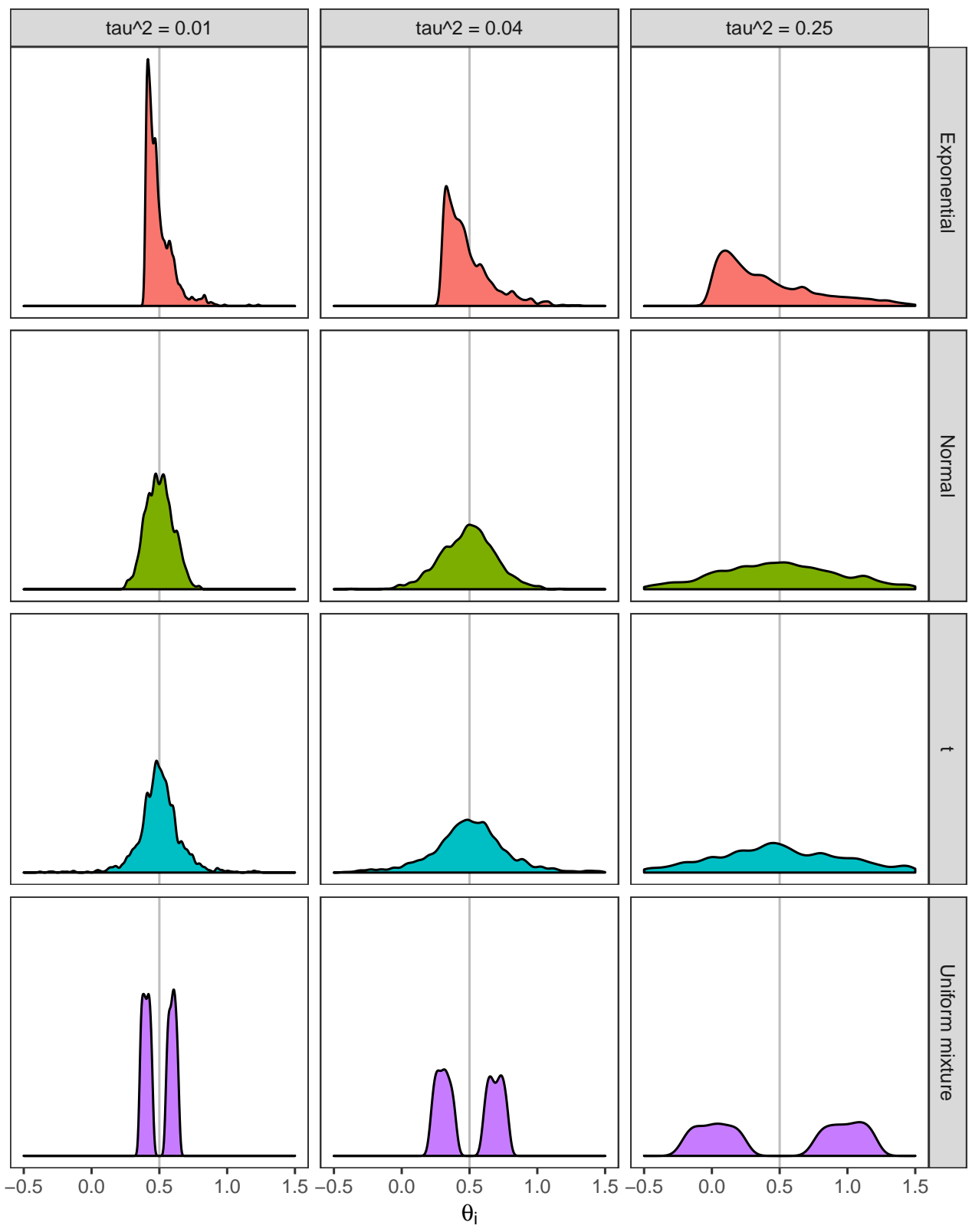

eFigure 1: Distributions of true population effects $\left(\theta_{i}\right)$ used in simulation study for varying choices of heterogeneity

\subsection{Summary of results}

Tables 2 and 3 summarize the performance of $95 \%$ confidence intervals and of point estimates respectively. For clarity given the very large number of simulation scenarios, the tables summarize results according to a single varied simulation parameter that produced particularly interesting variation in performance across methods (i.e., $\tau^{2}$ for inference and the true effect 
distribution for point estimation). Comprehensive simulation results for all 480 scenarios are also presented in the figures of Sections 2.3,2.6, these results are also publicly available as a dataset (https://osf.io/6nyg8/).

\begin{tabular}{ccccc}
\hline$\tau^{2}$ & Method & Coverage & $\begin{array}{c}\text { Minimum } \\
\text { coverage }\end{array}$ & Width \\
\hline \multirow{2}{*}{0.01} & BCa-calibrated & 0.99 & 0.90 & 0.52 \\
& BCa-parametric & 0.94 & 0.62 & 0.50 \\
& Parametric & 0.84 & 0.50 & 0.36 \\
& Sign test & 0.90 & 0.16 & 0.37 \\
\hline \multirow{2}{*}{0.04} & BCa-calibrated & 0.98 & 0.91 & 0.38 \\
& BCa-parametric & 0.89 & 0.44 & 0.35 \\
& Parametric & 0.82 & 0.45 & 0.28 \\
& Sign test & 0.96 & 0.72 & 0.33 \\
\hline \multirow{2}{*}{0.25} & BCa-calibrated & 0.98 & 0.92 & 0.32 \\
& BCa-parametric & 0.86 & 0.33 & 0.28 \\
& Parametric & 0.82 & 0.39 & 0.25 \\
& Sign test & 0.97 & 0.84 & 0.31 \\
\hline
\end{tabular}

eTable 2: Performance of $95 \%$ confidence intervals for scenarios with $k \geq 10$, showing mean coverage, minimum coverage, and mean width of $95 \%$ confidence intervals aggregating all scenarios with a given amount of heterogeneity $\left(\tau^{2}\right)$. 


\begin{tabular}{ccccc}
\hline Distribution & Method & RMSE & Bias & Absolute bias \\
\hline \multirow{3}{*}{ Exponential } & Calibrated & 0.162 & 0.022 & 0.097 \\
& Parametric & 0.164 & 0.042 & 0.105 \\
& Sign test max. & 0.141 & 0.036 & 0.089 \\
\hline \multirow{2}{*}{ Normal } & Calibrated & 0.159 & 0.005 & 0.094 \\
& Parametric & 0.135 & 0.006 & 0.080 \\
& Sign test max. & 0.135 & 0.014 & 0.084 \\
\hline \multirow{4}{*}{ t } & Calibrated & 0.151 & 0.007 & 0.089 \\
& Parametric & 0.150 & 0.027 & 0.088 \\
& Sign test max. & 0.135 & 0.010 & 0.082 \\
\hline \multirow{2}{*}{ Uniform mixture } & Carametric & 0.135 & 0.007 & 0.083 \\
& Sign test max. & 0.137 & 0.015 & 0.087 \\
\hline
\end{tabular}

eTable 3: Performance of methods for point estimation, showing means across all scenarios for each distribution of root mean squared error (RMSE), bias, and absolute bias. 
2.3. All coverage results by distribution
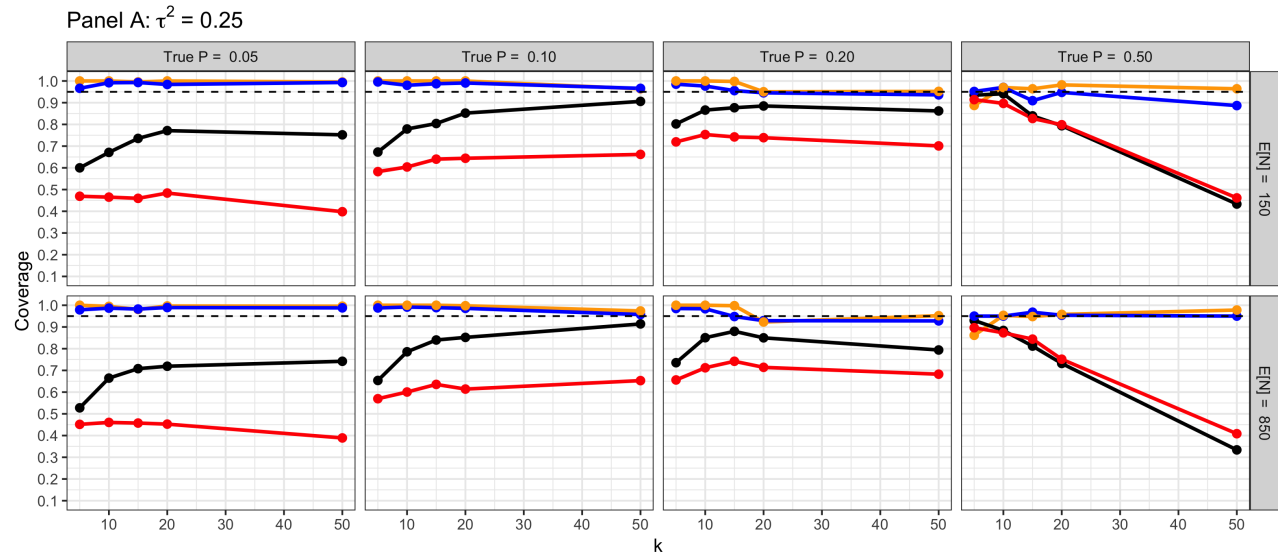

Method

$\sim$ BCa-calibrated

- BCa-parametric

$\rightarrow$ Parametric

- Sign test
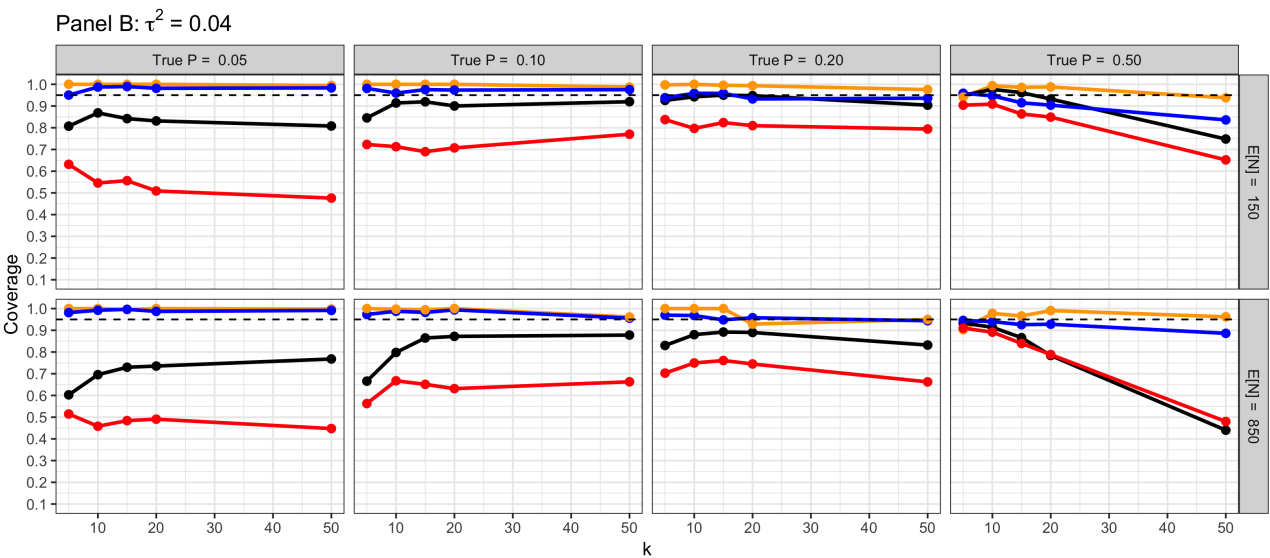

\section{Method}

$\sim$ BCa-calibrated

$\rightarrow$ BCa-parametric

- Parametric

— Sign test
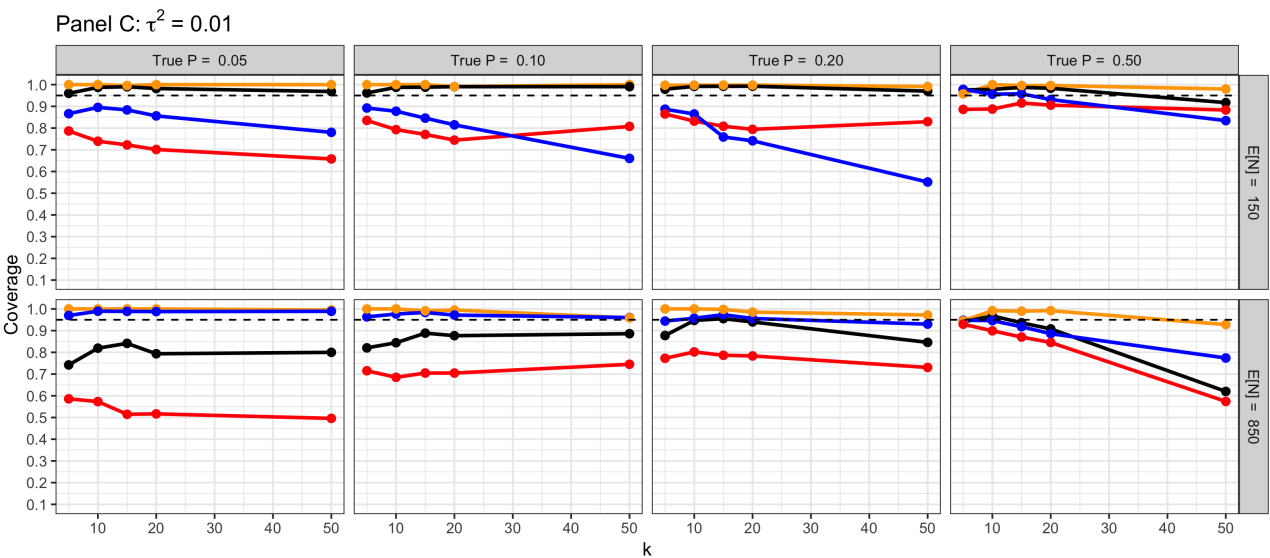

Method

- BCa-calibrated

$\rightarrow$ BCa-parametric

- Parametric

$\rightarrow$ Sign test

eFigure 2: Coverage of $95 \%$ confidence intervals for exponential distribution 

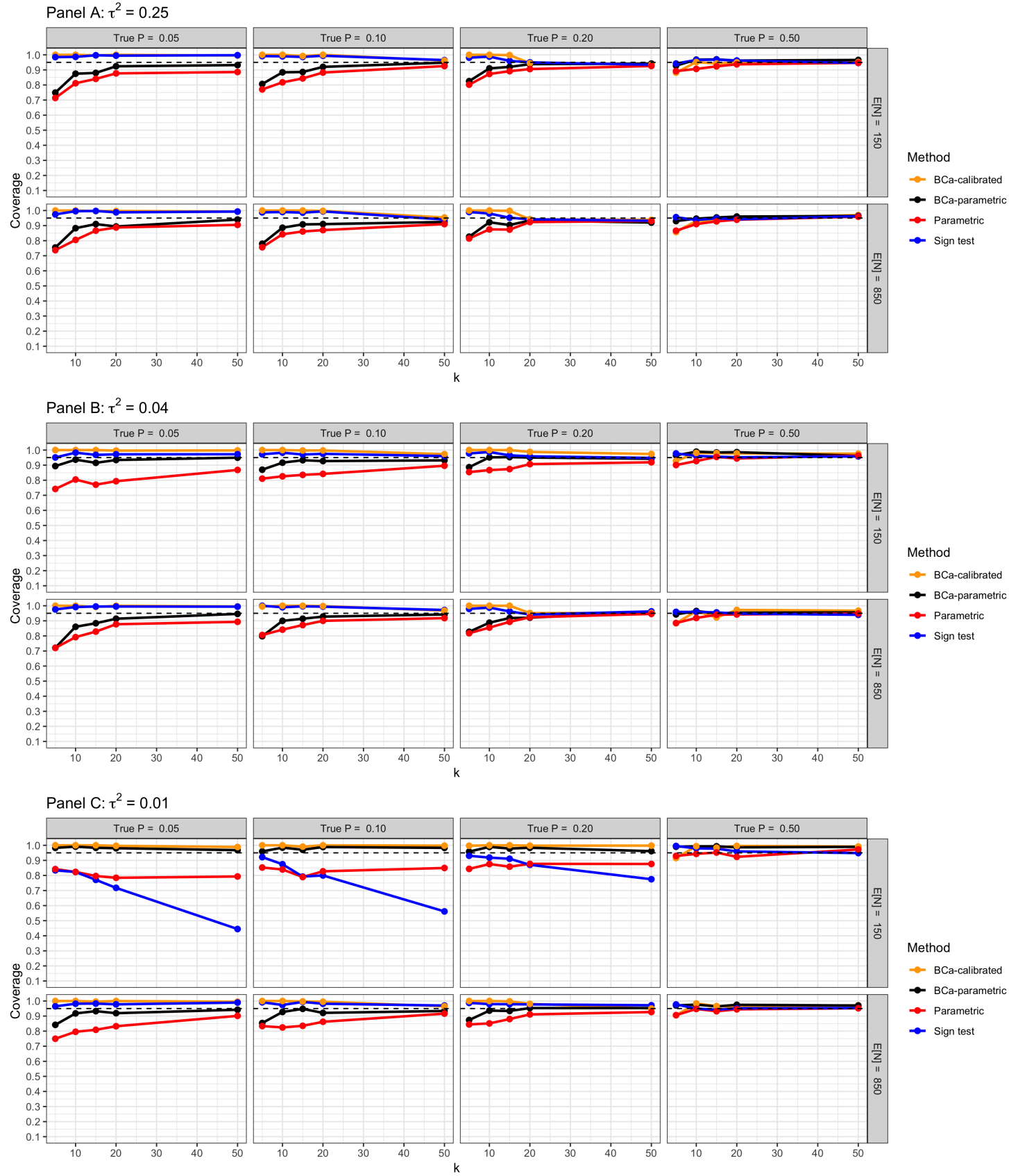

eFigure 3: Coverage of 95\% confidence intervals for normal distribution 

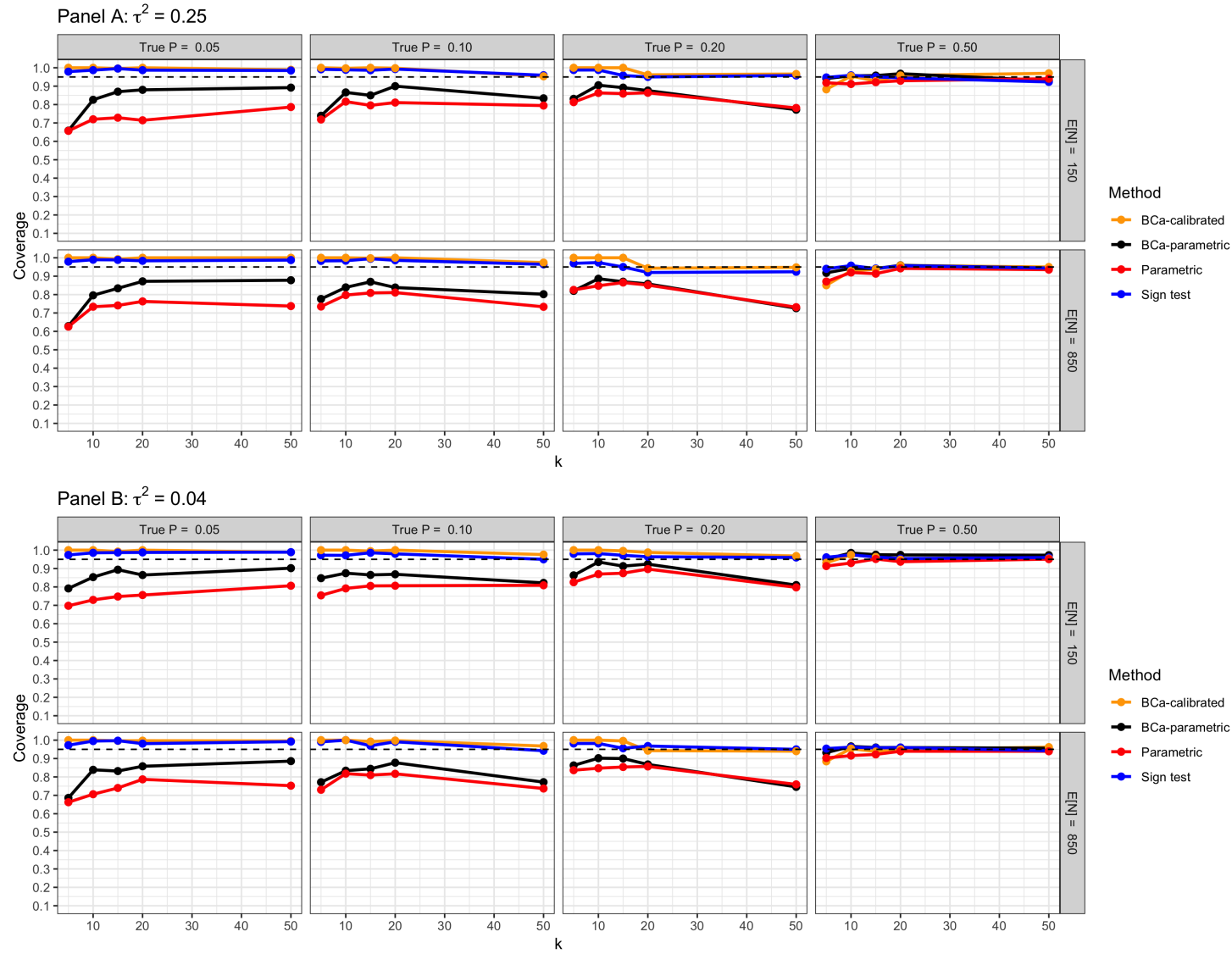

Method

$\sim$ BCa-calibrated

$\rightarrow$ BCa-parametric

$\rightarrow$ Parametric

$\rightarrow$ Sign test
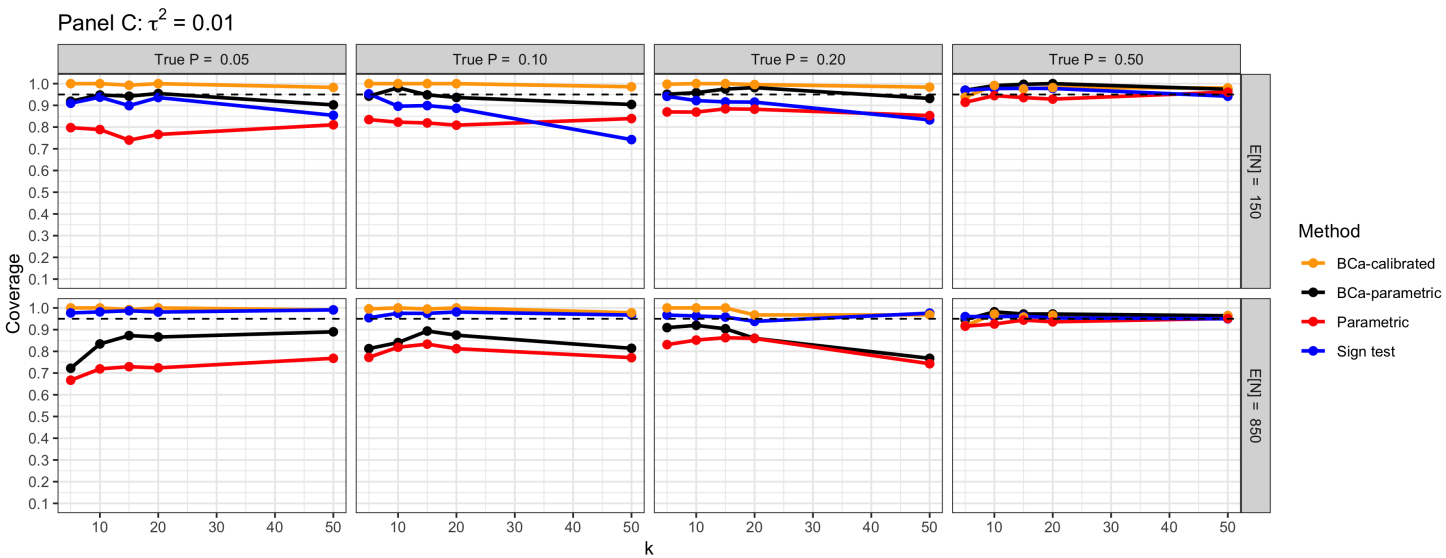

eFigure 4: Coverage of 95\% confidence intervals for scaled $t$ distribution 

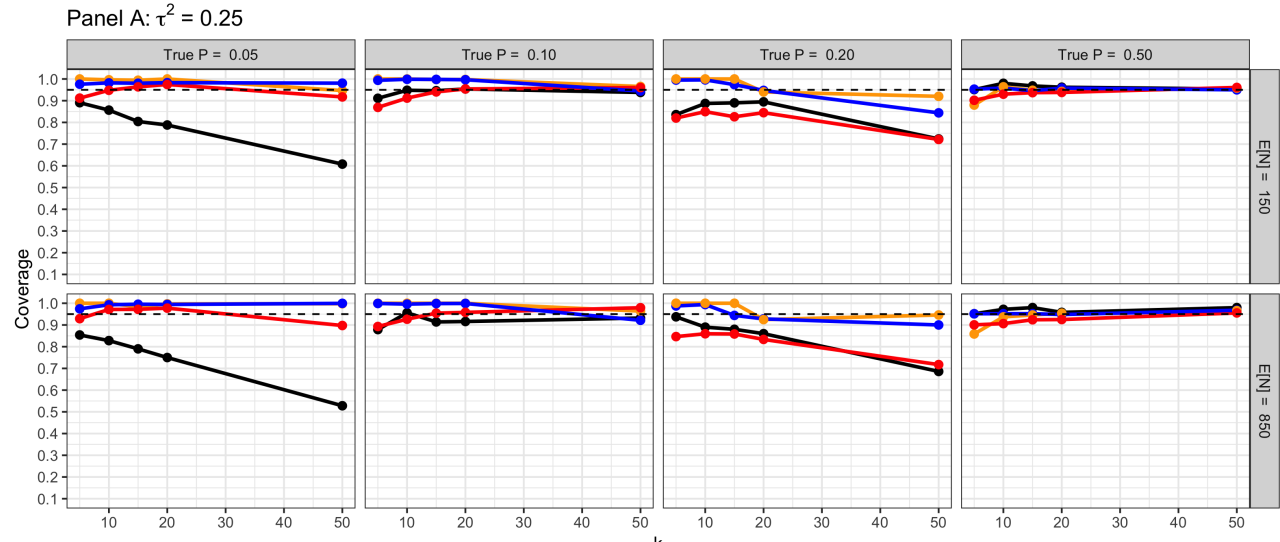

Method

$\sim$ BCa-calibrated

$\sim$ BCa-parametric
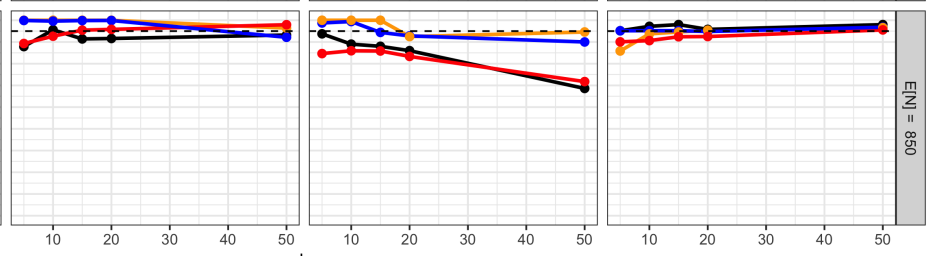

- Parametric

- Sign test
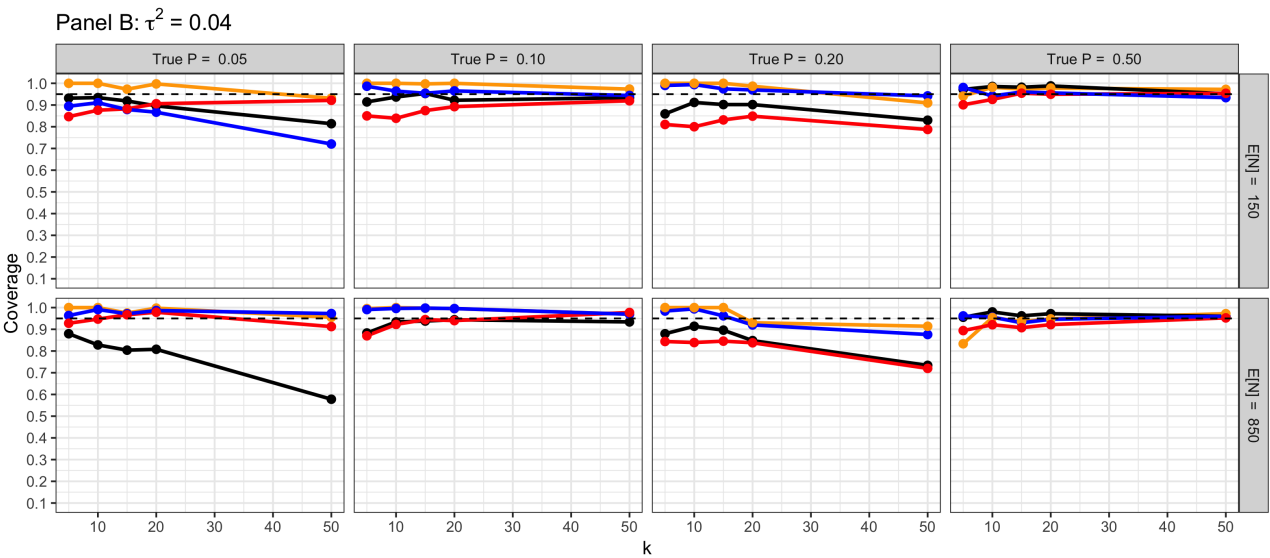

Method

$\rightarrow$ BCa-calibrated

- BCa-parametric

- Parametric

$\rightarrow$ Sign test
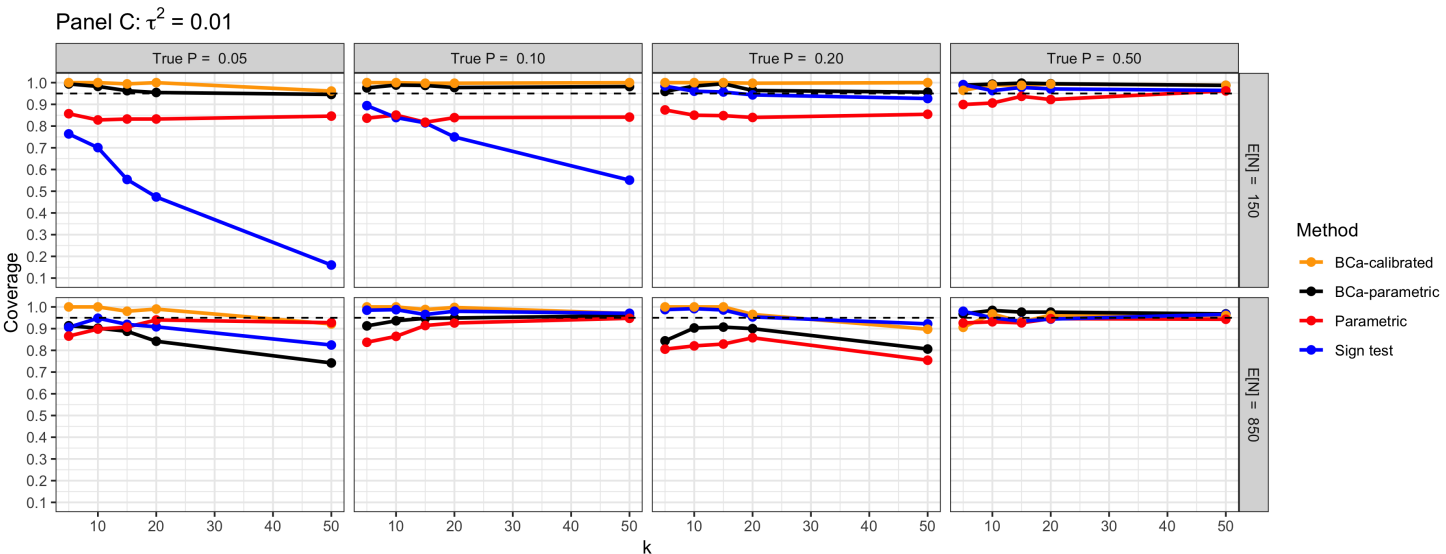

eFigure 5: Coverage of 95\% confidence intervals for uniform mixture distribution 
2.4. All confidence interval width results by distribution
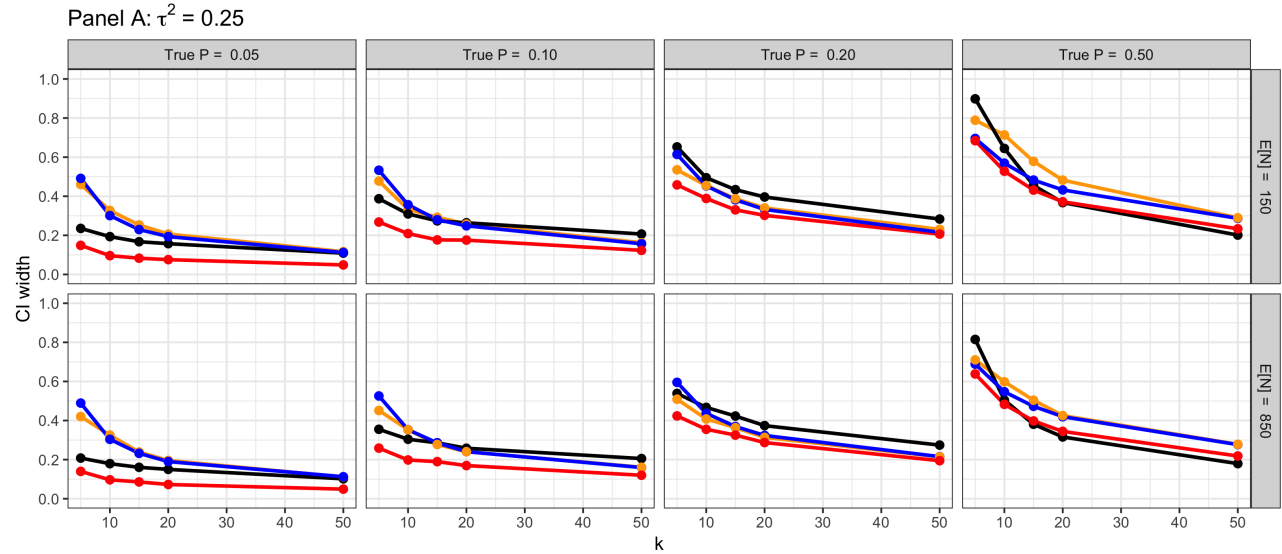

Method

$\sim$ BCa-calibrated

- BCa-parametric

$\rightarrow$ Parametric

- Sign test
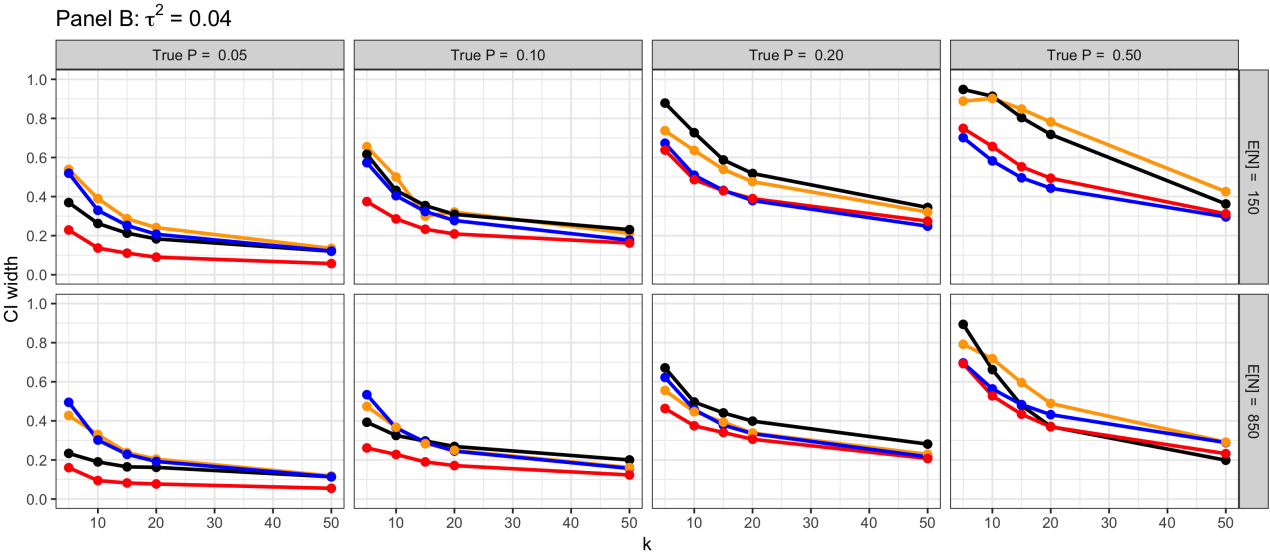

Method

$\sim$ BCa-calibrated

$\rightarrow$ BCa-parametric

- Parametric

$\sim$ Sign test
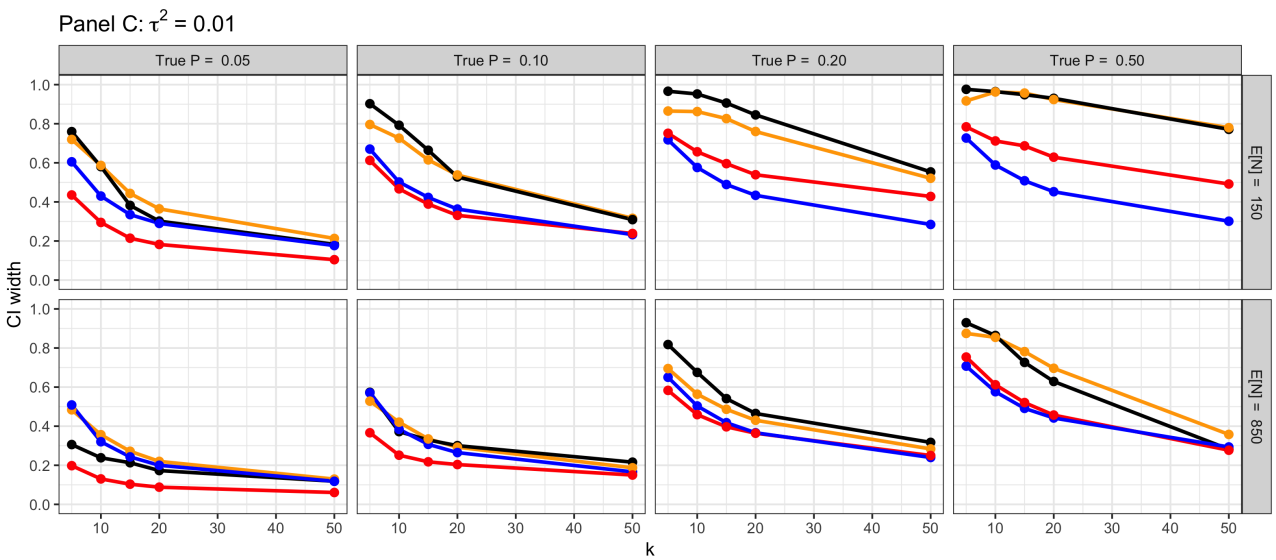

Method

$\approx$ BCa-calibrated

$\rightarrow$ BCa-parametric

- Parametric

$\rightarrow$ Sign test

eFigure 6: Width of 95\% confidence intervals for exponential distribution 

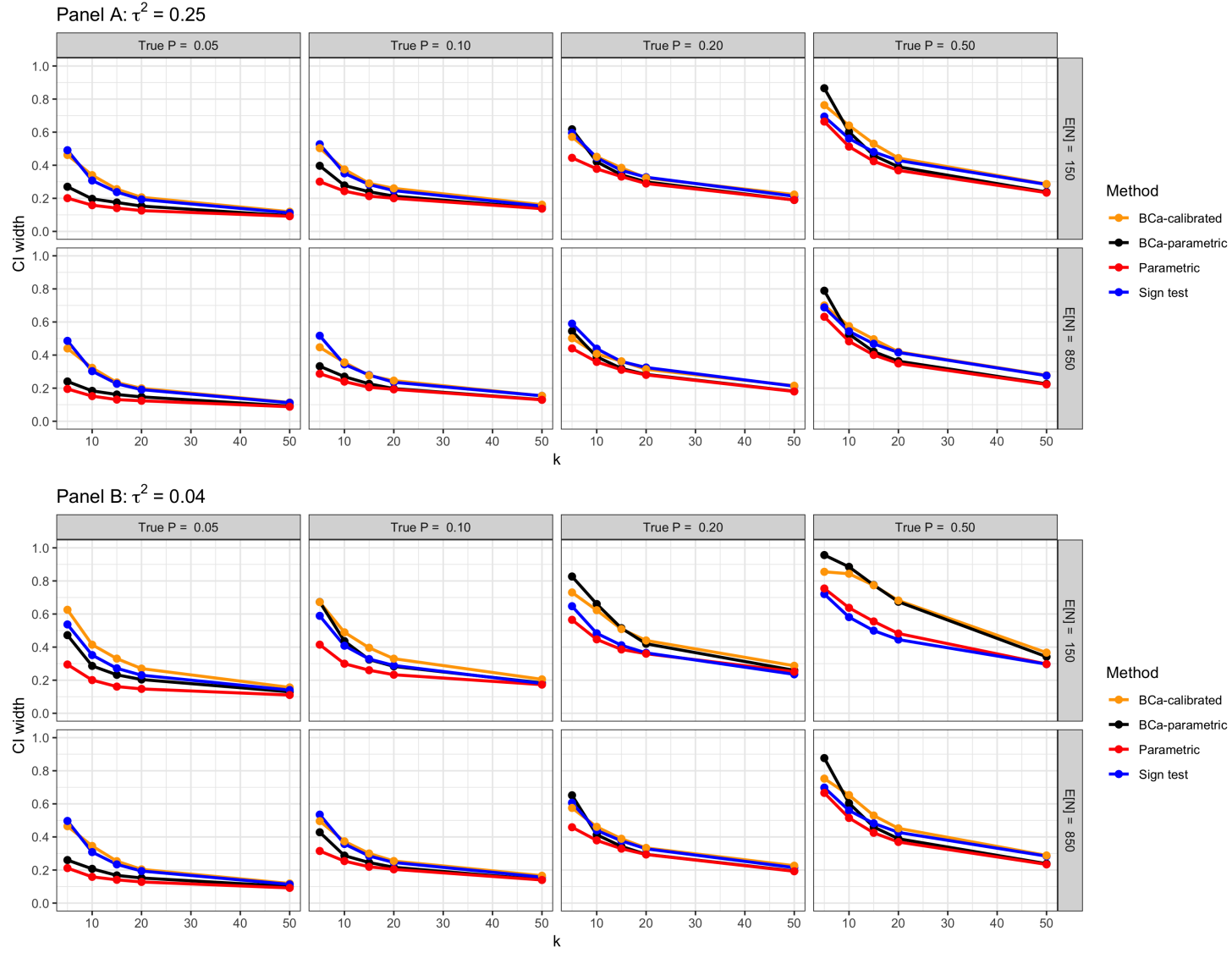

Method

$\sim$ BCa-calibrated

$\rightarrow$ BCa-parametric

$\rightarrow$ Parametric

- Sign test
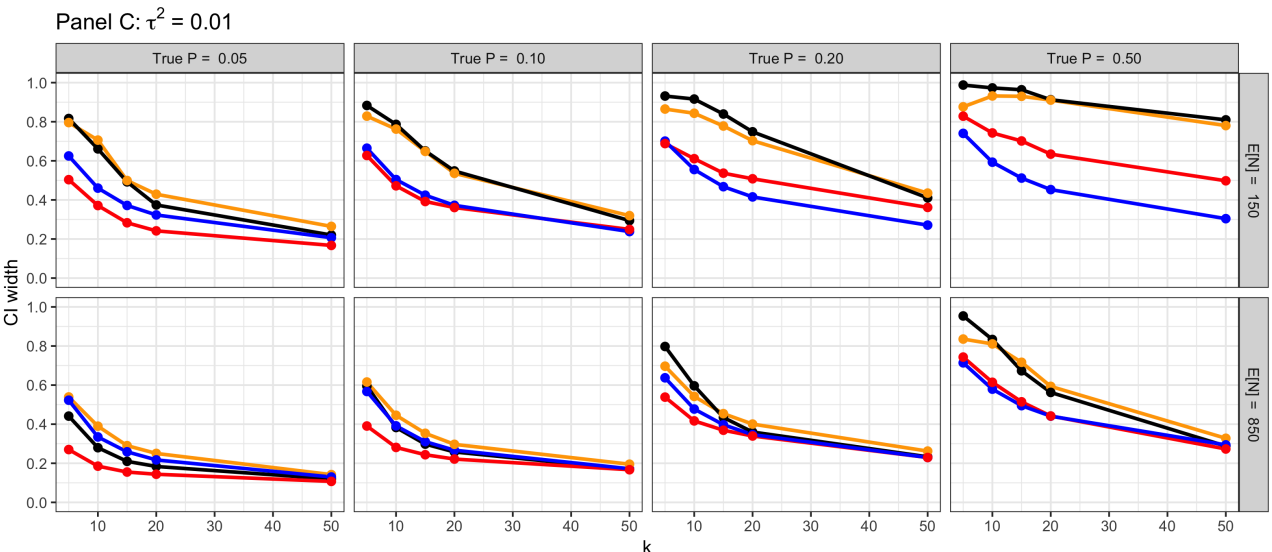

Method

$\rightarrow$ BCa-calibrated

$\rightarrow$ BCa-parametric

$\rightarrow$ Parametric

$\rightarrow$ Sign test

eFigure 7: Width of 95\% confidence intervals for normal distribution 

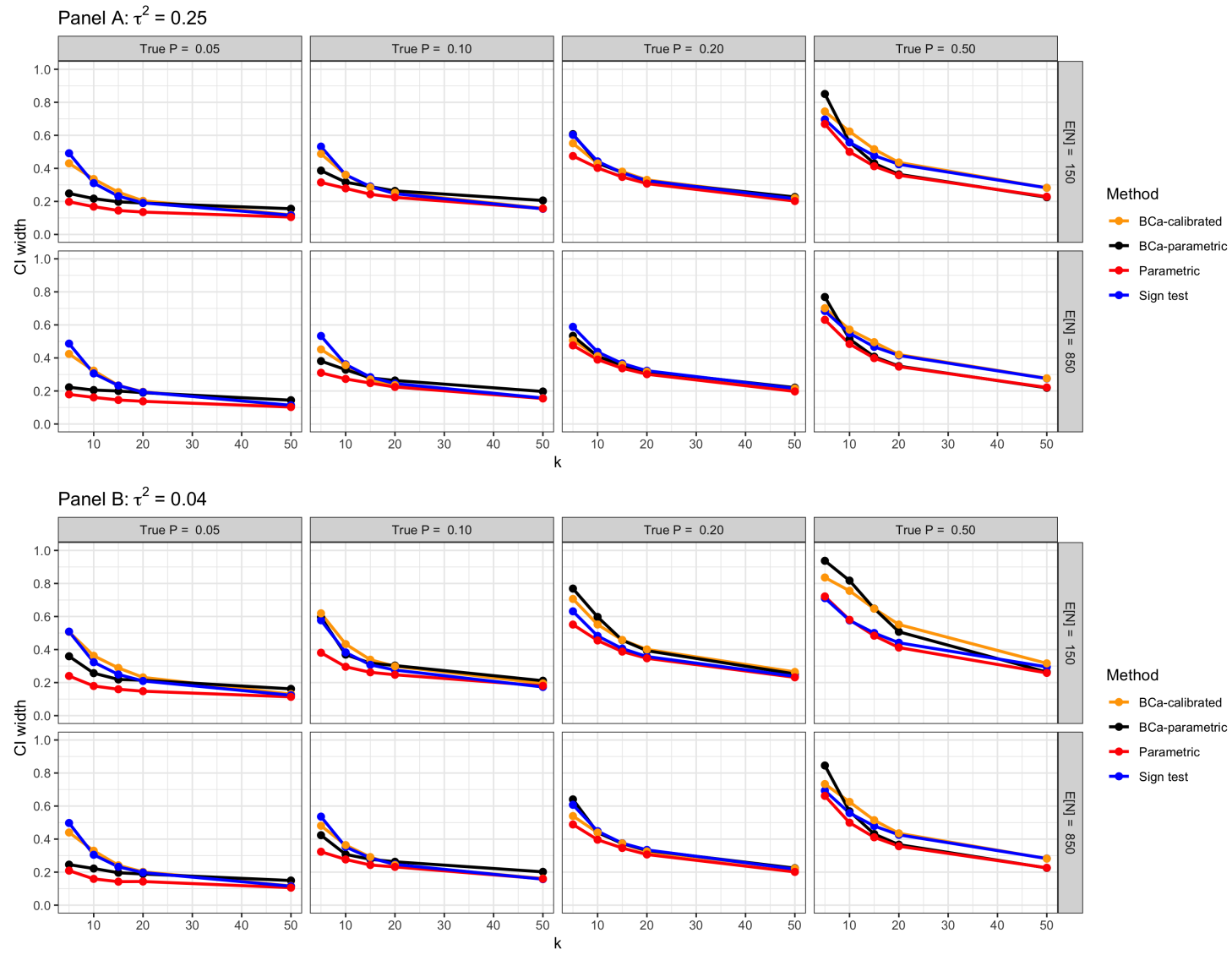

Method

$\sim$ BCa-calibrated

$\rightarrow$ BCa-parametric

$\rightarrow$ Parametric

- Sign test
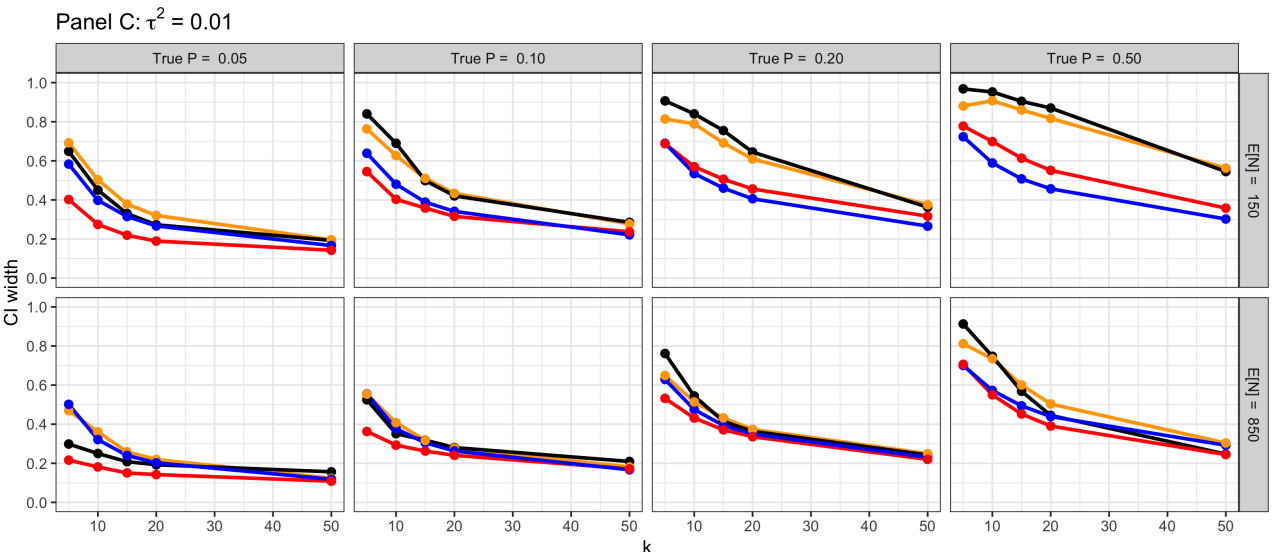

Method

$\rightarrow$ BCa-calibrated

$\rightarrow$ BCa-parametric

$\rightarrow$ Parametric

$\rightarrow$ Sign test

eFigure 8: Width of 95\% confidence intervals for scaled $t$ distribution 

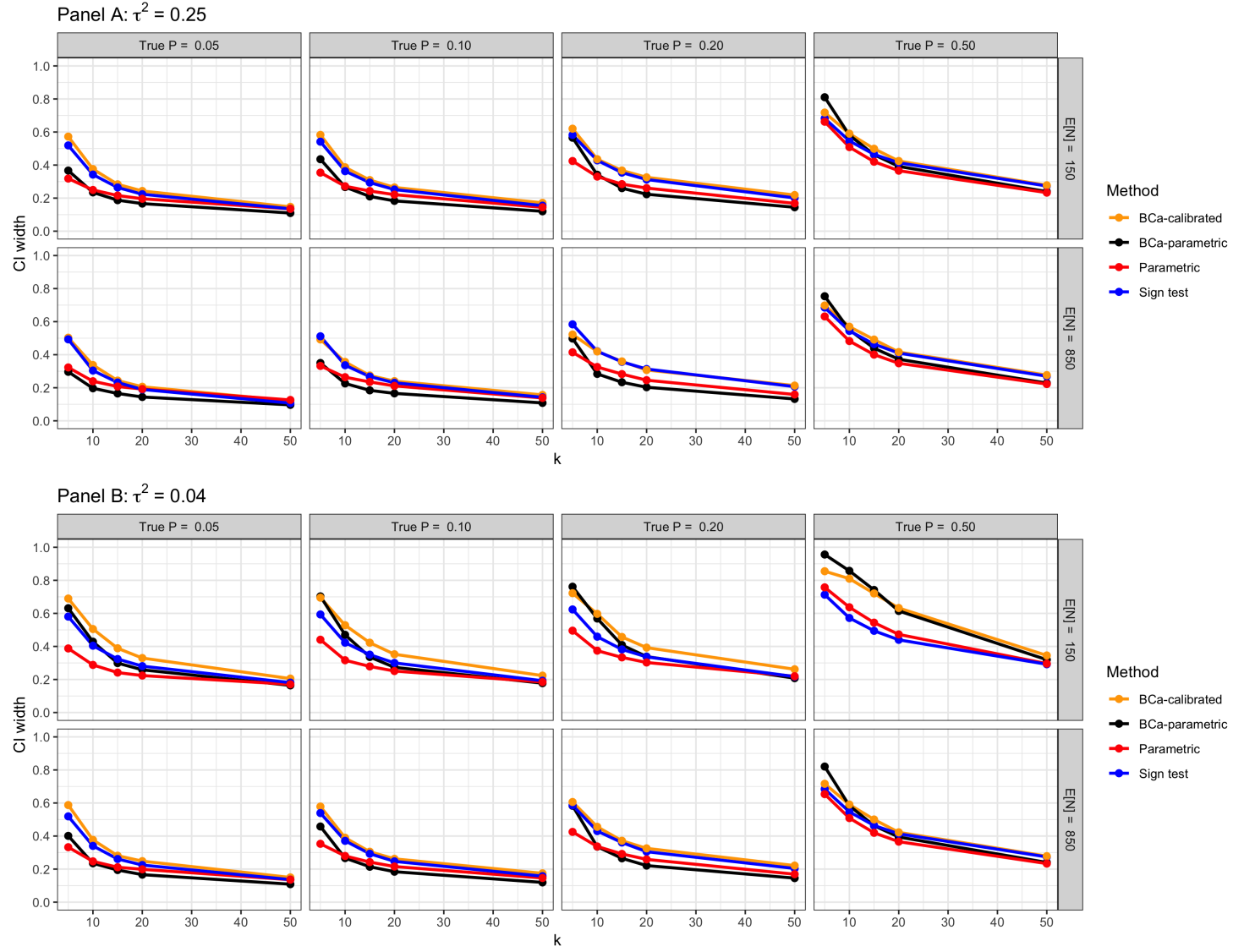

Method

$\sim$ BCa-calibrated

$\rightarrow$ BCa-parametric

$\rightarrow$ Parametric

- Sign test
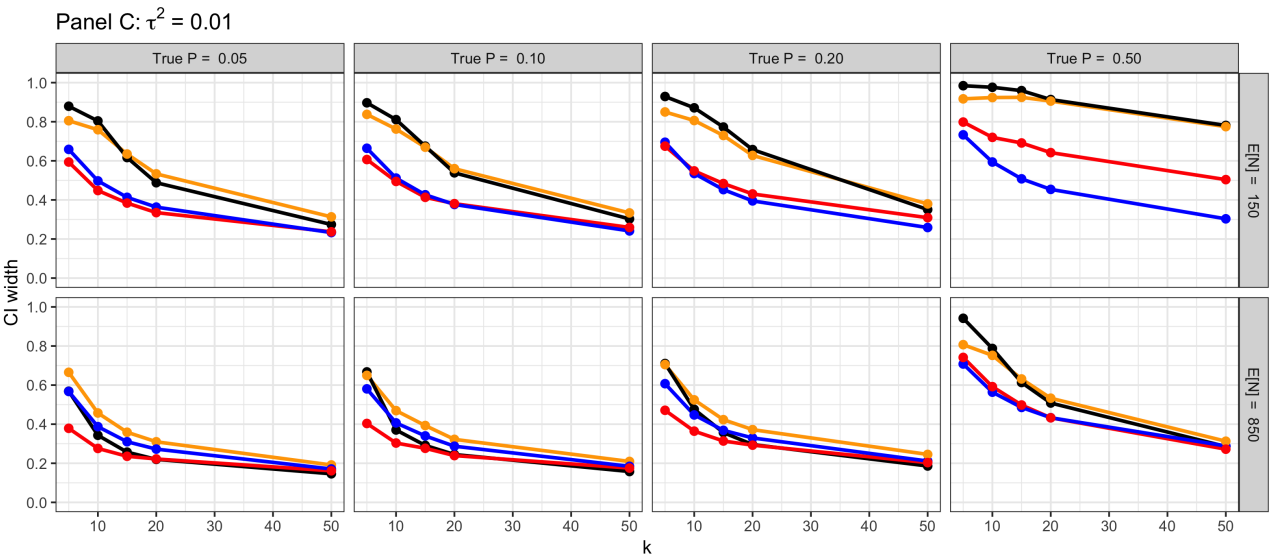

Method

$\rightarrow$ BCa-calibrated

$\rightarrow$ BCa-parametric

$\rightarrow$ Parametric

$\rightarrow$ Sign test

eFigure 9: Width of 95\% confidence intervals for uniform mixture distribution 
2.5. All RMSE results by distribution
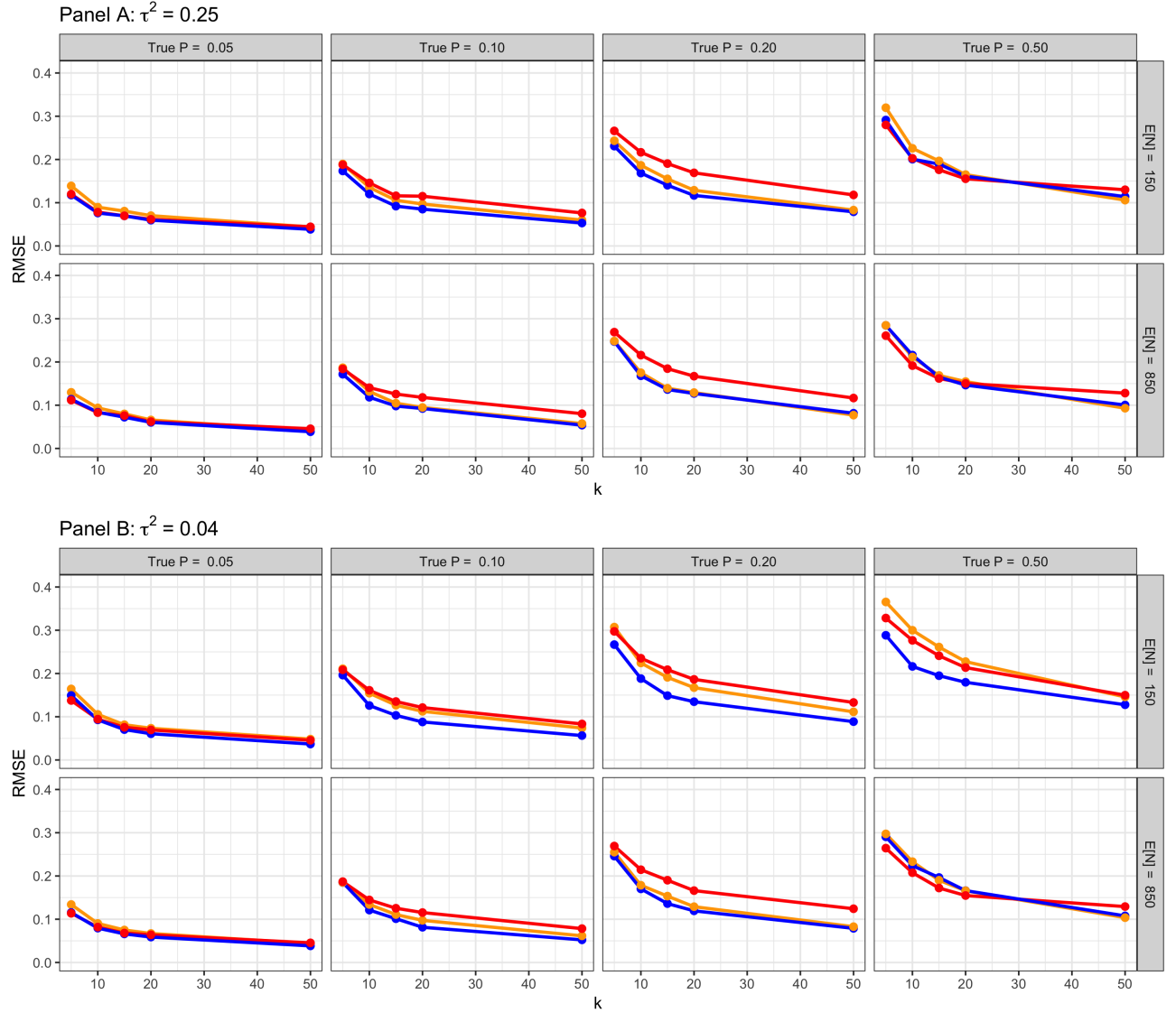

Method

$\rightarrow$ Calibrated

- Parametric

- Sign test max
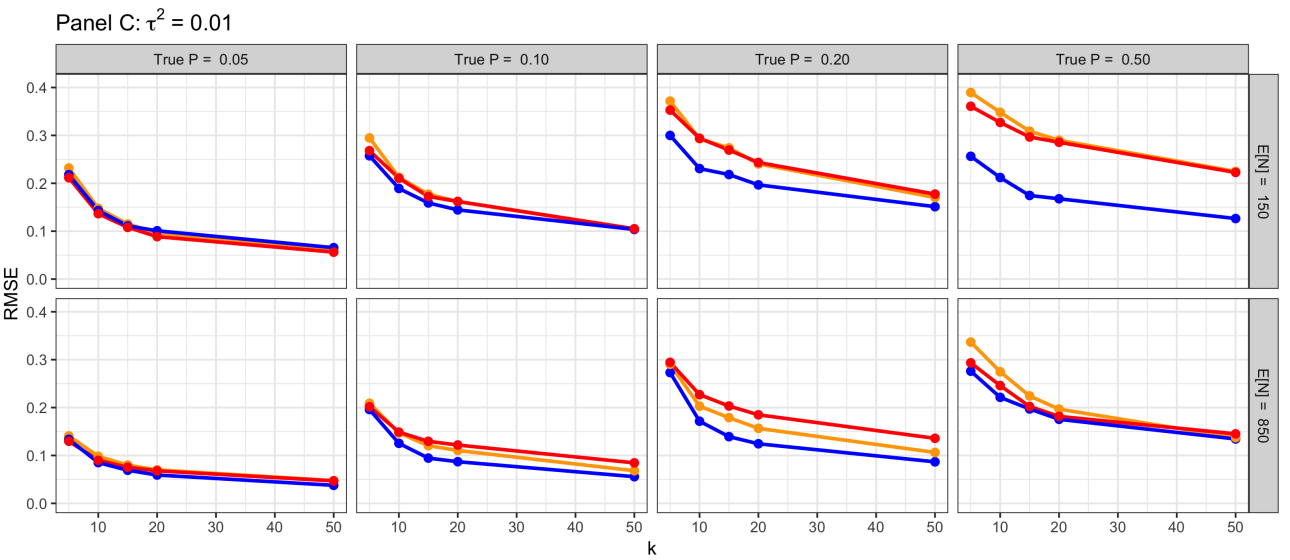

Method

- Calibrated

- Parametric

- Sign test max

eFigure 10: RMSE of point estimates for exponential distribution

Method

- Calibrated

$\rightarrow$ Parametric

— Sign test max. 

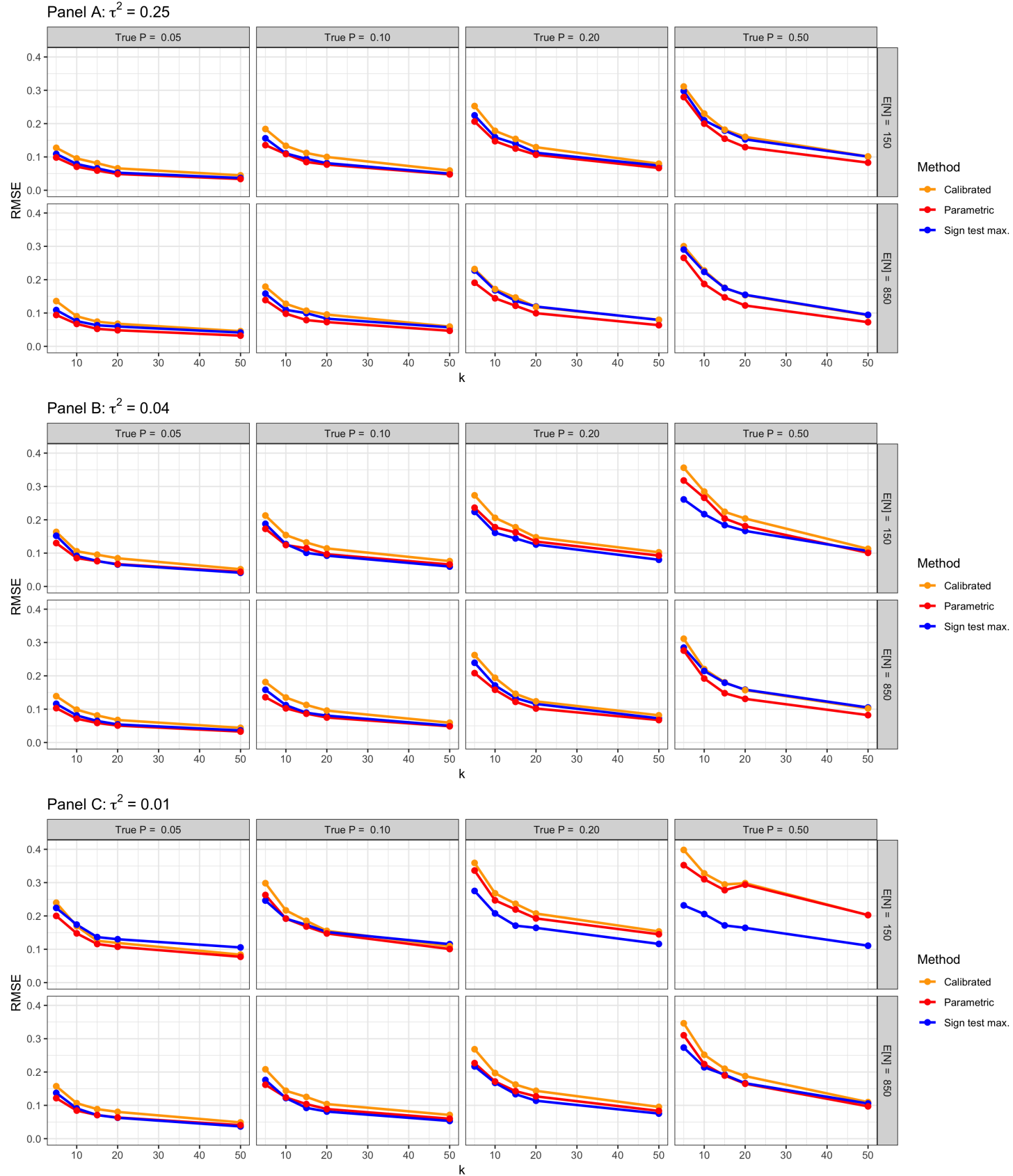

eFigure 11: RMSE of point estimates for normal distribution 

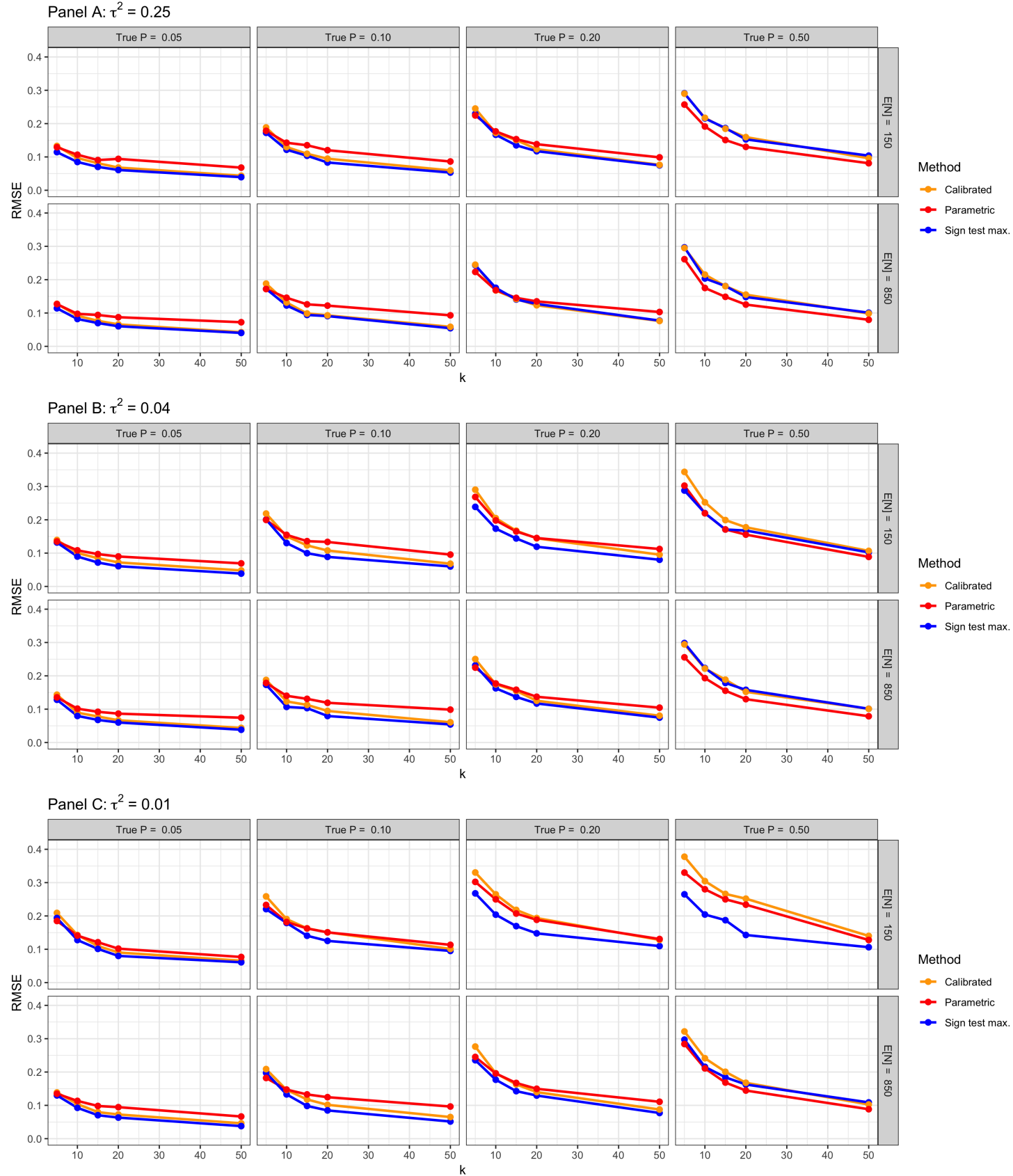

eFigure 12: RMSE of point estimates for scaled $t$ distribution 

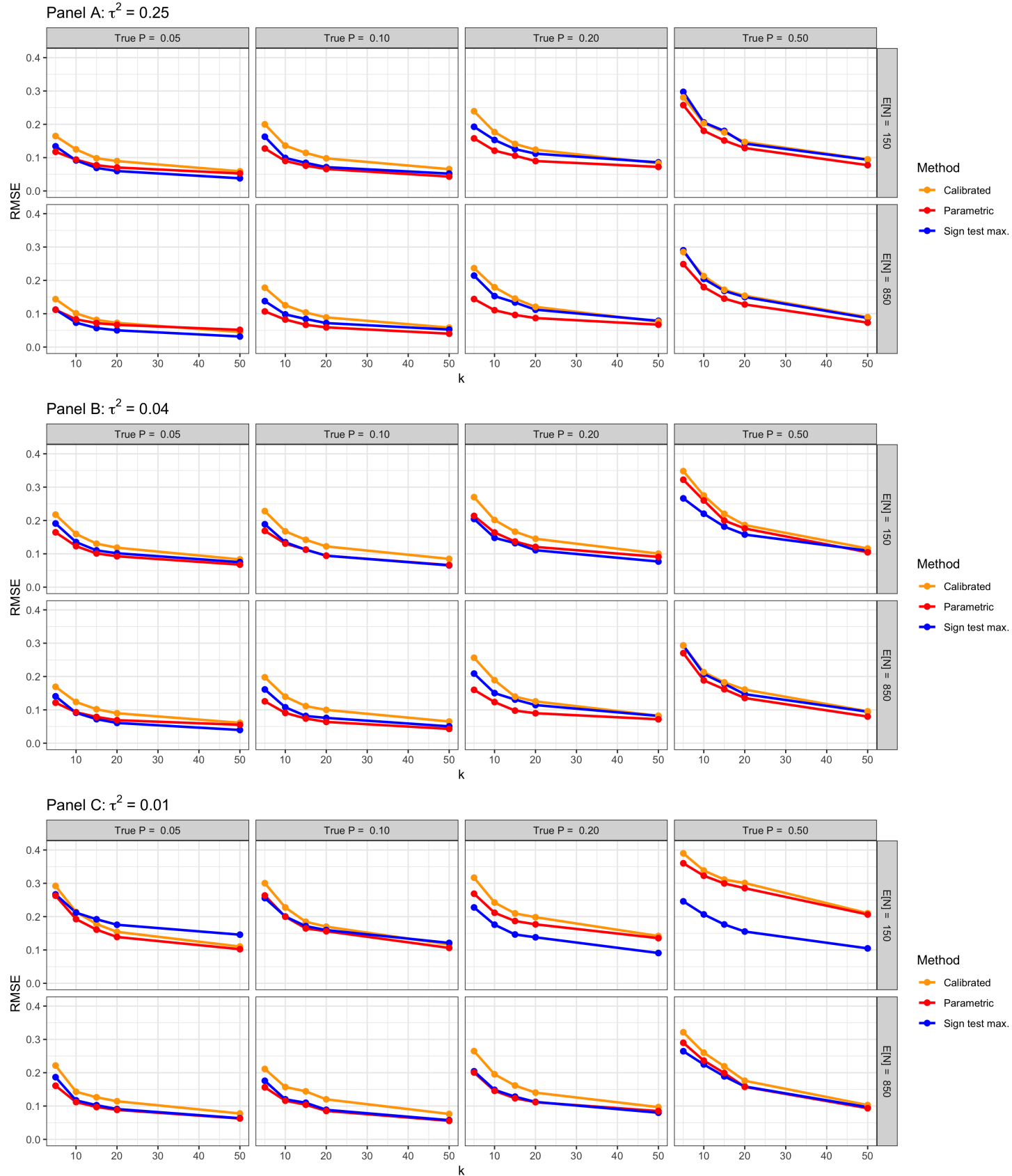

eFigure 13: RMSE of point estimates for uniform mixture distribution 
2.6. All bias results by distribution
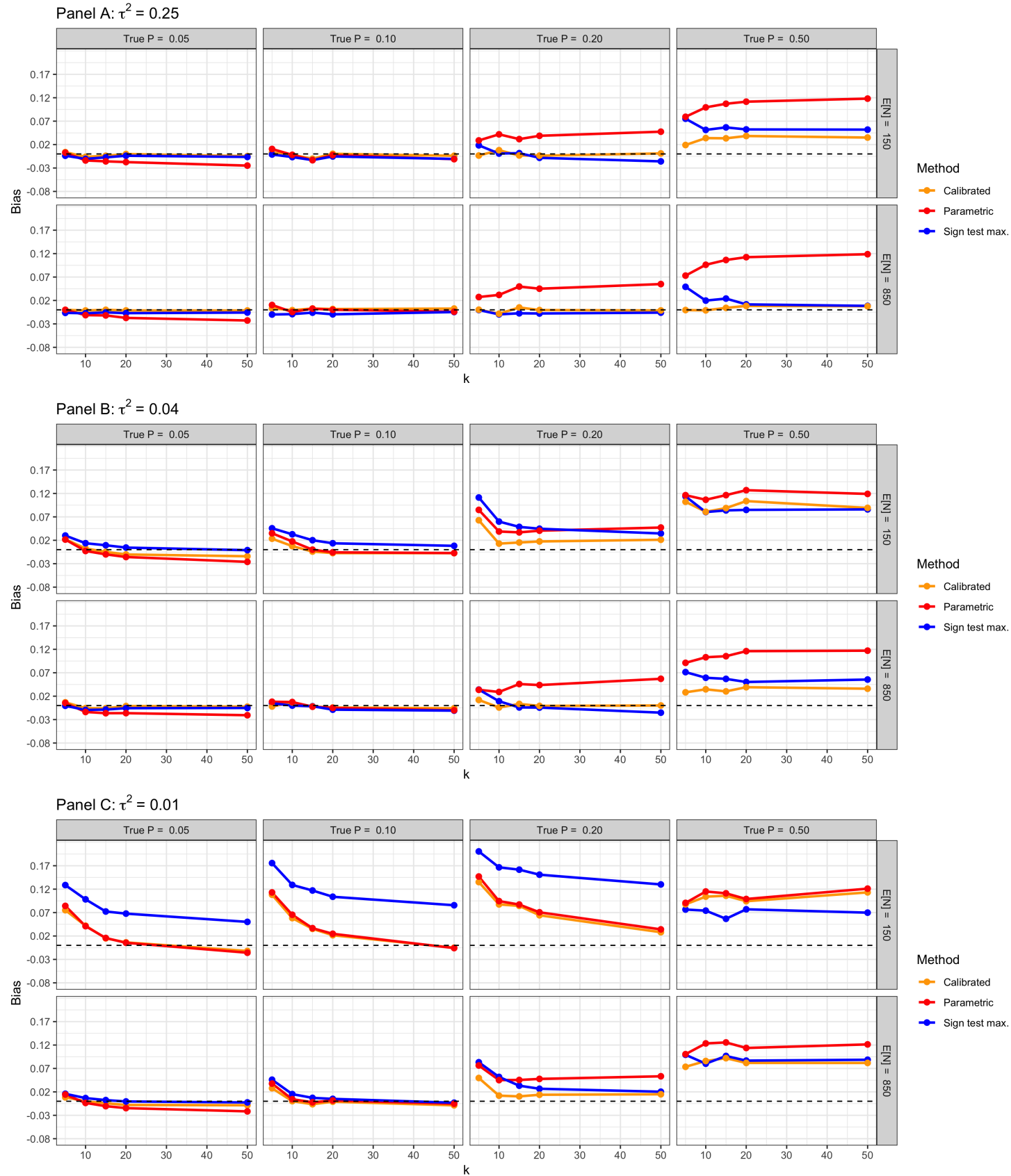

eFigure 14: Bias of point estimates for exponential distribution 

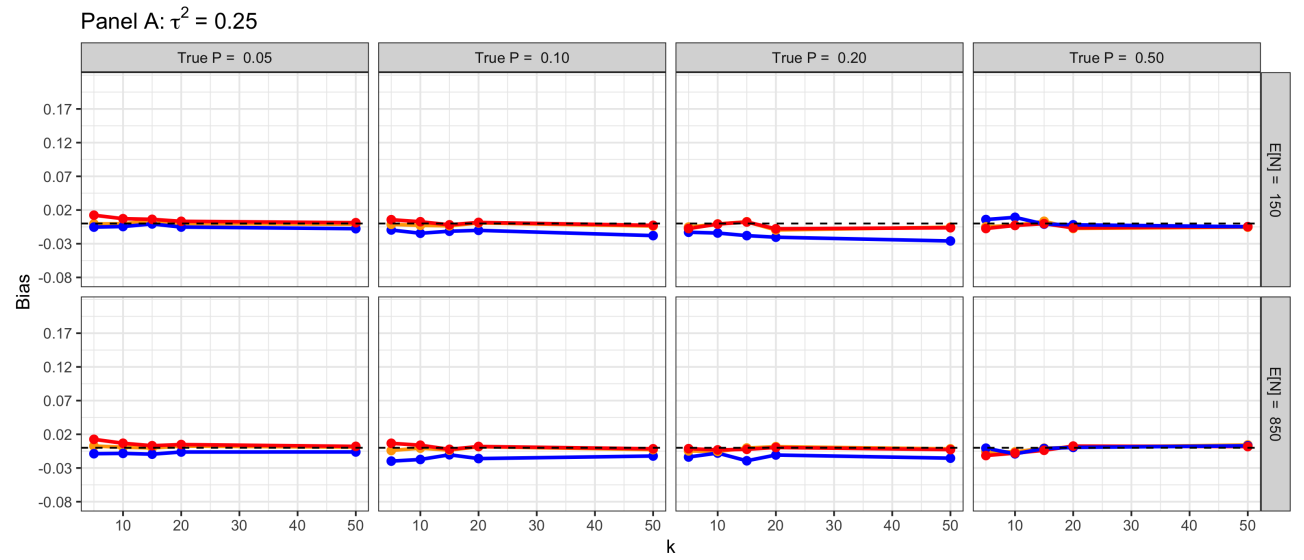

Method

- Calibrated

$\rightarrow$ Parametric

- Sign test max
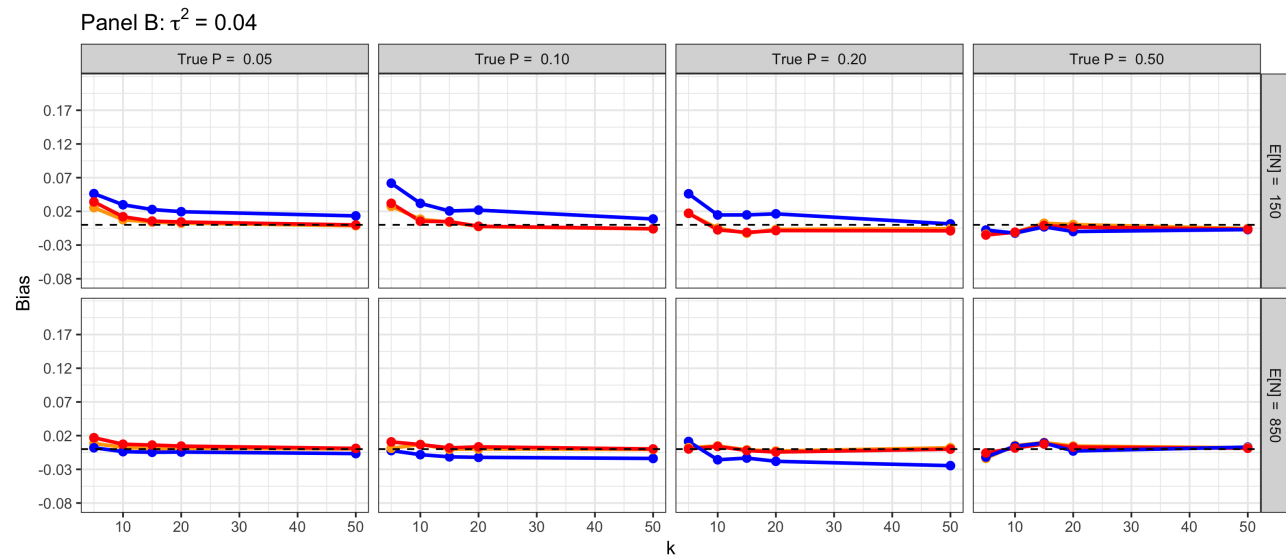

Method

$\sim$ Calibrated

$\sim$ Parametric

$\rightarrow$ Sign test max.
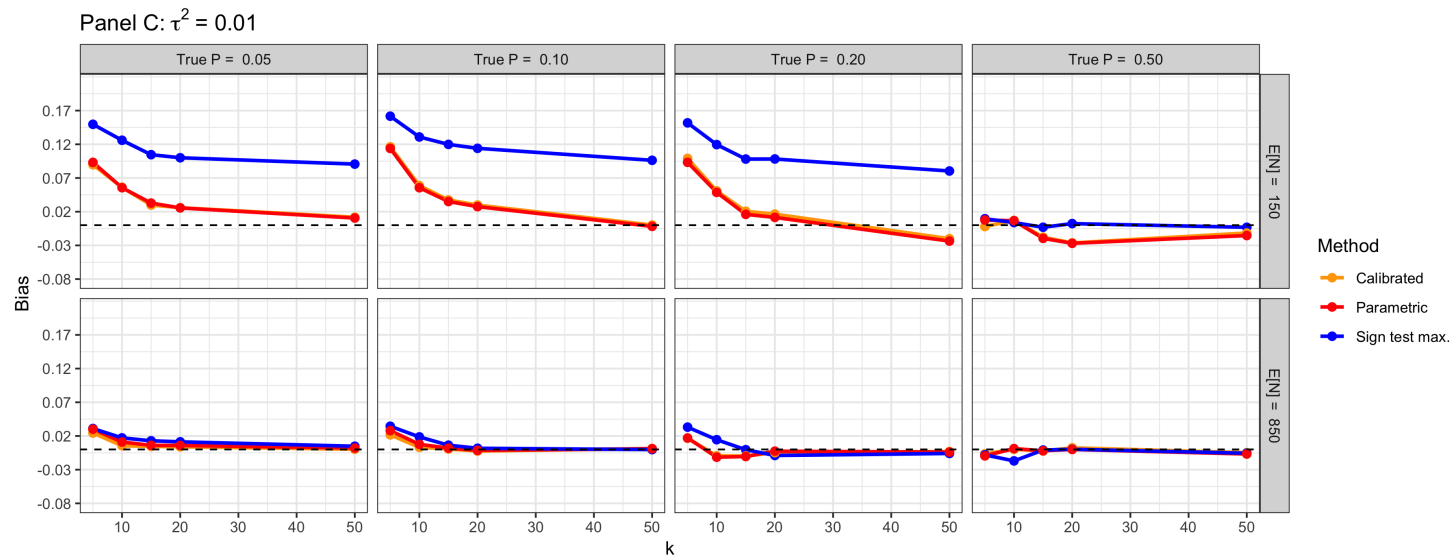

eFigure 15: Bias of point estimates for normal distribution 

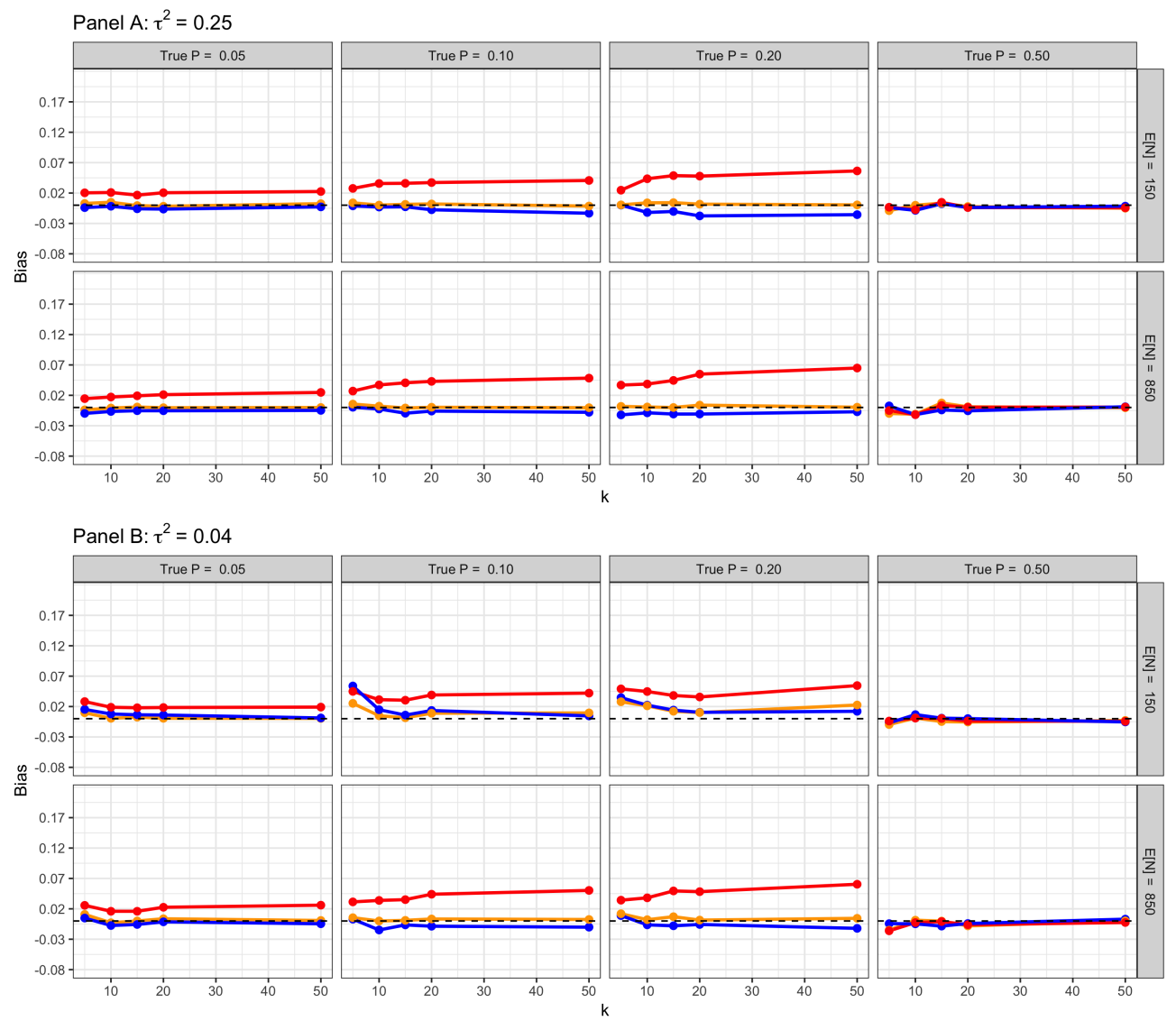

Method

- Calibrated

- Parametric

- Sign test max
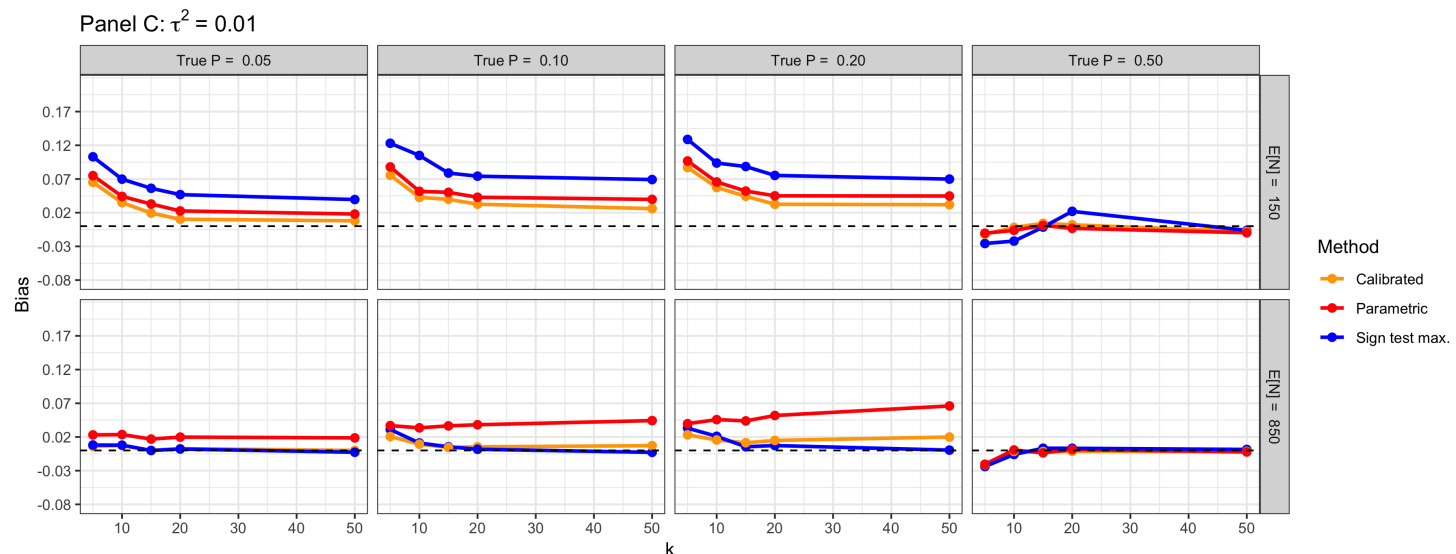

eFigure 16: Bias of point estimates for scaled $t$ distribution 

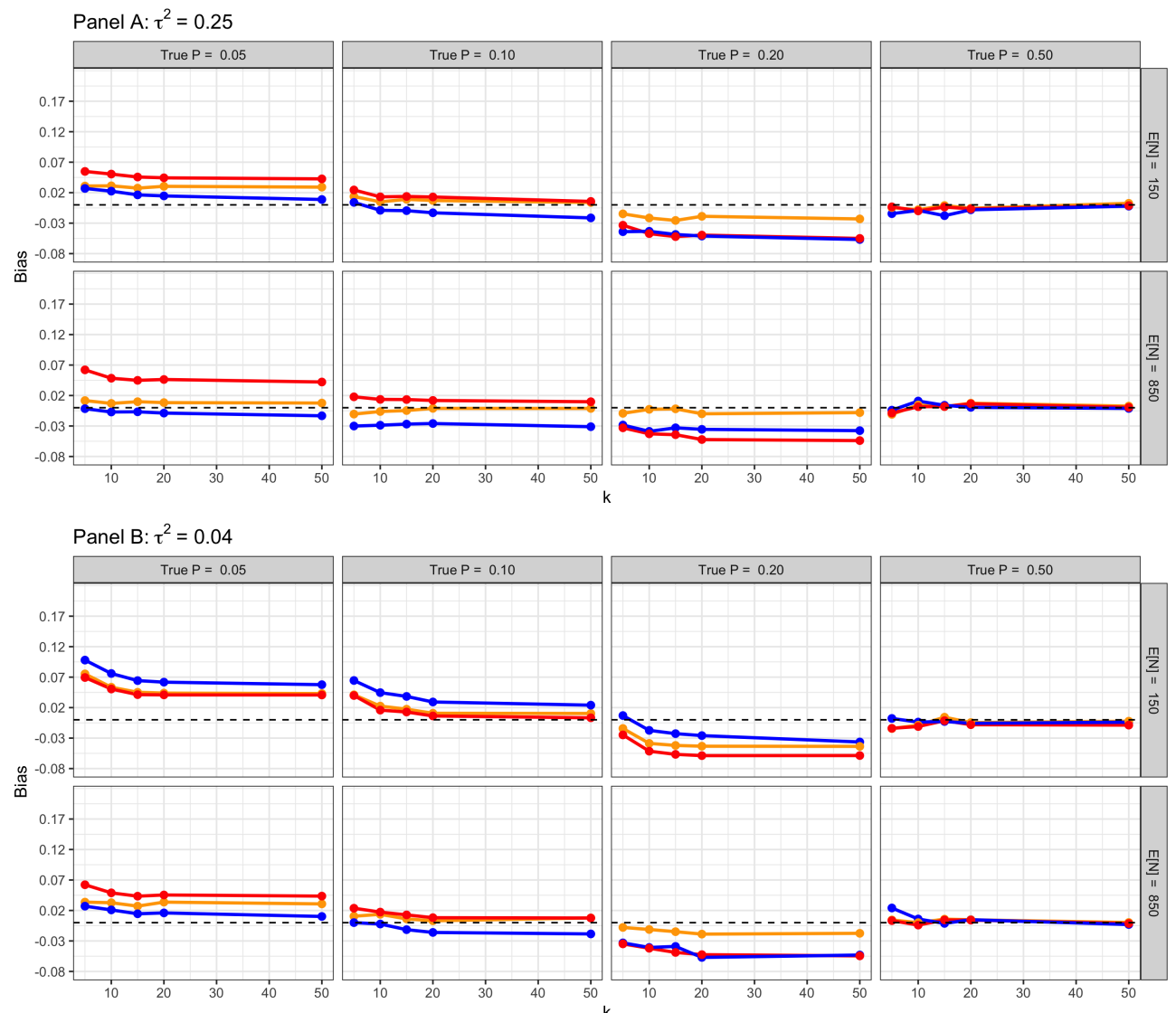

Method

- Calibrated

$\sim$ Parametric

$\sim$ Sign test max.
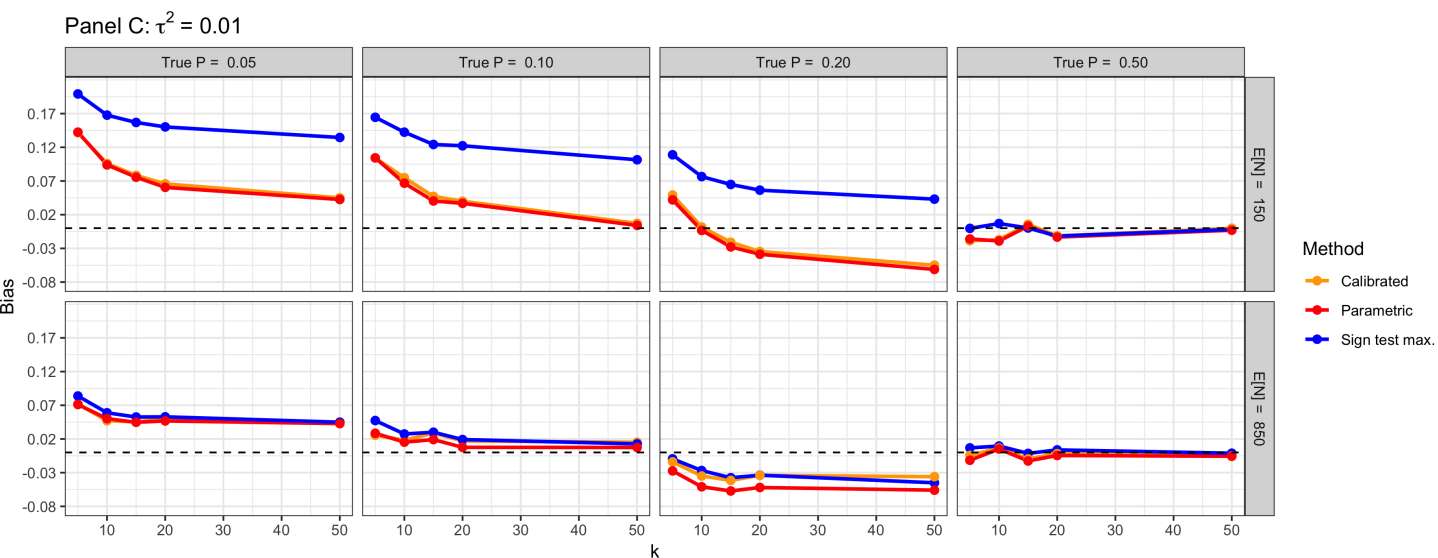

eFigure 17: Bias of point estimates for uniform mixture distribution 


\section{APPLIED EXAMPLE}

We illustrate the methods using two meta-analyses of randomized controlled trials on the effect of mass deworming programs in developing countries on children's bodyweights. A Cochrane meta-analysis ${ }^{9}$ of 11 studies reported "little to no effect" (estimated mean increase in bodyweight [kg]: 0.08; 95\% CI: [-0.11, 0.27]), while an updated meta-analysis of 22 studies by other investigators ${ }^{100}$ reported "significant" mean increases in weight (estimate: 0.13;95\% CI: $[0.03,0.24])$. A largely unresolved controversy ensued ${ }^{[11]}$. We obtained data for both meta-analyses $\frac{109}{109}$ from Croke et al.'s (2016) ${ }^{100}$ Figures 1 and 2; the data are reproduced in Figures 18a and 18b. (We fit random-effects meta-analysis models using restricted maximum likelihood with standard errors adjusted via the Knapp-Hartung method in keeping with best practices ${ }^{1213}$; hence, the confidence intervals reported in our analyses are slightly wider than those the original authors reported using the Dersimonian-Laird method ${ }^{10}$, which are the estimates reported above.) Figure 19 shows estimated densities of the standardized point estimates, $\left(\widehat{\theta}_{i}-\widehat{\mu}\right) / \sqrt{\widehat{\tau}^{2}+\widehat{\sigma}_{i}^{2}}$, in each meta-analysis ${ }^{\sqrt{14}}$ and suggests some non-normality in both cases. Similarly, Shapiro-Wilk tests on the standardized point estimates yielded $p=0.11$ and $p=0.02$ for the Taylor-Robinson et al. (2015) respectively, also suggesting some non-normality 15 .

We therefore used the calibrated estimates to estimate the proportions of effects in each meta-analysis above and below several effect size thresholds, and we used the BCa-calibrated method for confidence intervals. For all thresholds, the meta-analyses in fact seemed to agree closely (Table 4). For example, both suggested that a majority of effects are above $0\left(\widehat{P}_{>0}\right.$ $=0.73$ with $95 \%$ CI: $[0,1]$ and 0.82 with $95 \%$ CI: [0.41, 0.91] respectively $\left.{ }^{910}\right)$; these point estimates suggest frequent beneficial effects of mass deworming, albeit possibly of very small size. The meta-analyses also both suggested that a sizable minority of effects are above 0.2 $\operatorname{kg}\left(\widehat{P}_{>0.2}=0.18\right.$ with $95 \% \mathrm{CI}:[0,0.55]$ and 0.23 with $95 \%$ CI: $\left.[0,0.41]\right)$, though the wide confidence intervals indicated that there was considerable uncertainty. The analysis also indicated that in at least some settings, the programs may in fact decrease bodyweight by at least $0.2 \mathrm{~kg}$ on average $\left(\widehat{P}_{<-0.2}=0.18\right.$ with $95 \% \mathrm{CI}$ : $[0,0.45]$ and 0.14 with $95 \%$ CI: $\left.[0,0.36]\right)$. Although the point estimates appear to agree closely, some of the confidence intervals for the first meta-analysis ${ }^{\mathbb{9}}$ are quite wide, indicating considerable uncertainty. Table 1 illustrates how these results compare to those obtained using parametric methods. 


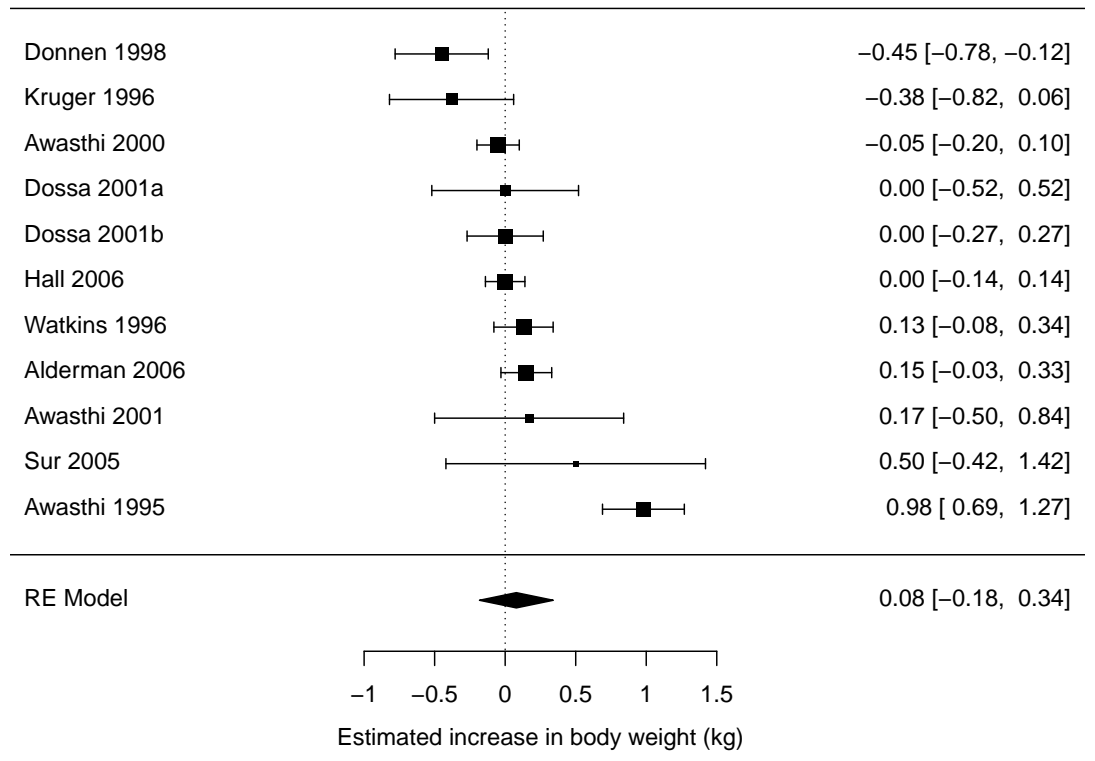

(a) Taylor-Robinson et al. (2015) $)^{\sqrt{9}}$ meta-analysis

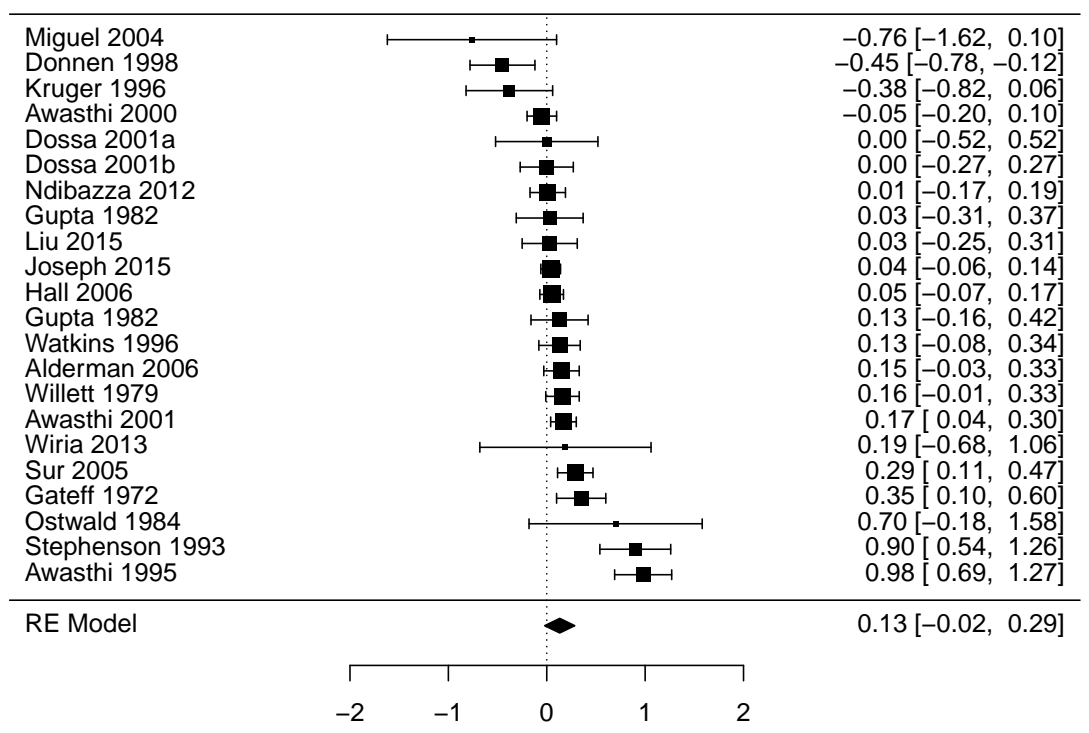

Estimated increase in body weight $(\mathrm{kg})$

(b) Croke et al. (2016) $\frac{10}{10}$ meta-analysis

eFigure 18: Forest plots for each meta-analysis of study-level point estimates (mean differences in bodyweight with vs. without mass deworming intervention) with $95 \%$ confidence intervals. Pooled point estimates were estimated via random-effects meta-analysis. 


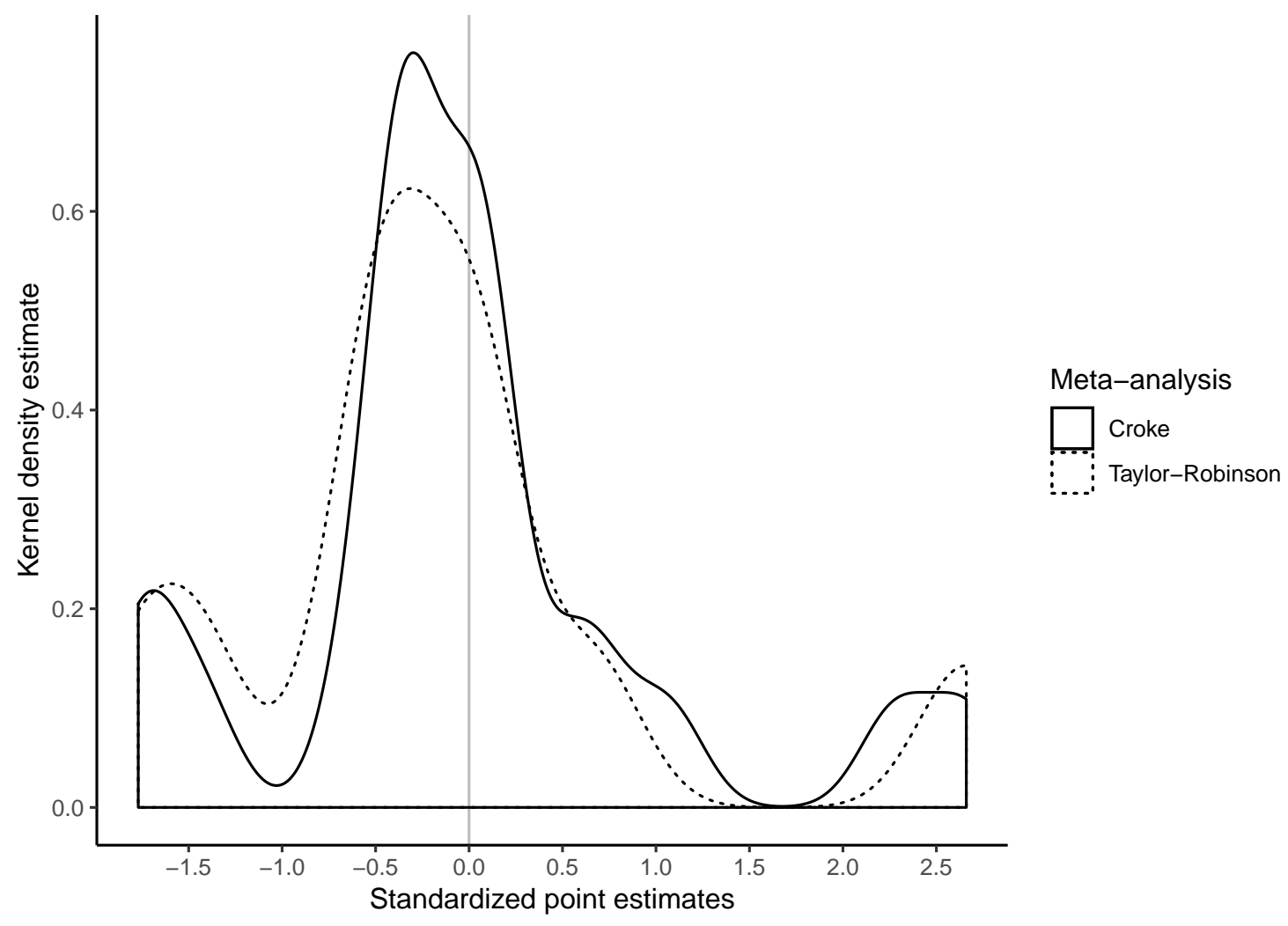

eFigure 19: Estimated densities of true population effects in each meta-analysis. 


\begin{tabular}{ccc}
\hline & Taylor-Robinson (2015) & Croke et al. (2016) \\
\hline$k$ & 11 & 22 \\
$\widehat{\mu}$ & $0.08[-0.18,0.34]$ & $0.13[-0.02,0.29]$ \\
$\widehat{\tau}$ & $0.35[0.00,0.51]$ & $0.28[0.13,0.38]$ \\
\hline
\end{tabular}

\begin{tabular}{ccccc} 
Est. \% of effects & Parametric & Calibrated & Parametric & Calibrated \\
$>0$ & $59[33,85]$ & $73[0,100]$ & $68[49,87]$ & $82[41,91]$ \\
$>0.1$ & $48[21,74]$ & $45[0,82]$ & $54[34,75]$ & $50[14,68]$ \\
$>0.2$ & $36[11,62]$ & $18[0,55]$ & $40[20,61]$ & $23[0,41]$ \\
$>0.5$ & $11[0,58]$ & $9[0,27]$ & $10[0,35]$ & $9[0,23]$ \\
$<-0.1$ & $30[5,55]$ & $18[0,36]$ & $21[3,38]$ & $14[0,27]$ \\
$<-0.2$ & $21[0,44]$ & $18[0,45]$ & $12[0,29]$ & $14[0,36]$ \\
\hline
\end{tabular}

eTable 4: Number of studies ( $k)$, pooled point estimates $(\widehat{\mu})$, heterogeneity estimates of standard deviation of true population effects $(\widehat{\tau})$, and estimated percent of estimates above and below various thresholds $\left(100 \% \times \widehat{P}_{>q}\right.$ or $\left.100 \% \times \widehat{P}_{<q}\right)$. "Parametric": point estimate was obtained parametrically and inference was obtained either via the delta method or by bootstrapping parametric estimates when the estimated percentage of effects was less than $15 \%$ or greater than $85 \%$. "Calibrated": point estimate and inference were obtained using the calibrated estimates and BCa bootstrapping. Effect sizes are presented on the raw mean difference scale ( $\mathrm{kg}$ of bodyweight). Brackets denote $95 \%$ confidence intervals.

\section{SOFTWARE}

The code below illustrates use of the function prop_stronger in the R package MetaUtility to estimate the proportion of effects above 0.5 in the Taylor-Robinson (2015) meta-analysis described above. The standard $\mathrm{R}$ documentation for the function provides details.

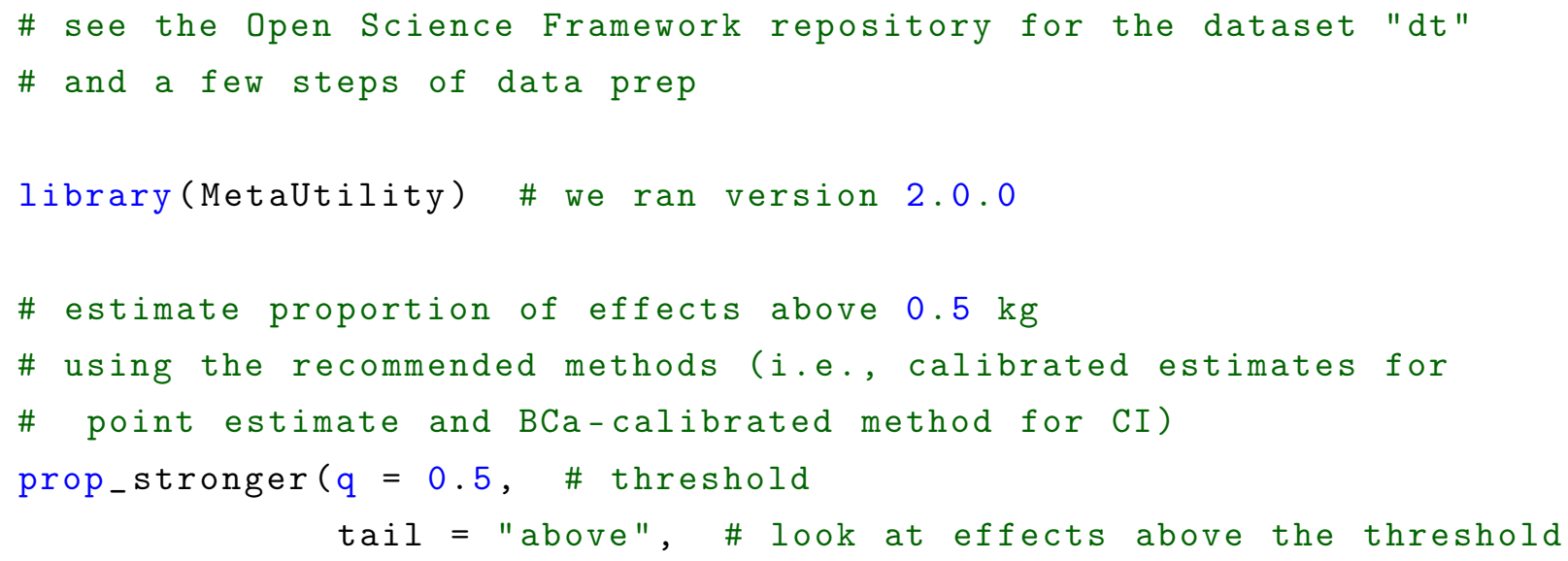




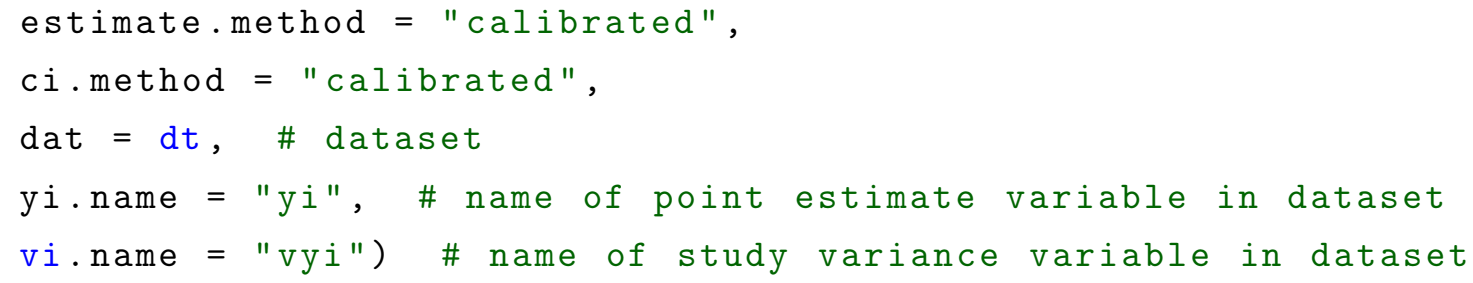

\section{REFERENCES}

[1] Rui Wang, Lu Tian, Tianxi Cai, and LJ Wei. Nonparametric inference procedure for percentiles of the random effects distribution in meta-analysis. The Annals of Applied Statistics, 4(1):520, 2010.

[2] Chia-Chun Wang and Wen-Chung Lee. A simple method to estimate prediction intervals and predictive distributions: Summarizing meta-analyses beyond means and confidence intervals. Research Synthesis Methods, 10(2):255-266, 2019.

[3] Maya B Mathur and Tyler J VanderWeele. Sensitivity analysis for unmeasured confounding in meta-analyses. Journal of the American Statistical Association, pages 1-20, 2019.

[4] Maya B Mathur and Tyler J VanderWeele. New metrics for meta-analyses of heterogeneous effects. Statistics in Medicine, 2018.

[5] Peng Ding and Tyler J VanderWeele. Sensitivity analysis without assumptions. Epidemiology (Cambridge, Mass.), 27(3):368, 2016.

[6] Tyler VanderWeele and Peng Ding. Sensitivity analysis in observational research: introducing the E-value. Annals of Internal Medicine, pages doi: 10.7326/M16-2607, 2017.

[7] Julian PT Higgins, Simon G Thompson, Jonathan J Deeks, and Douglas G Altman. Measuring inconsistency in meta-analyses. BMJ, 327(7414):557-560, 2003.

[8] Jeffrey C Valentine, Therese D Pigott, and Hannah R Rothstein. How many studies do you need? A primer on statistical power for meta-analysis. Journal of Educational and Behavioral Statistics, 35(2):215-247, 2010.

[9] David C Taylor-Robinson, Nicola Maayan, Karla Soares-Weiser, Sarah Donegan, and Paul Garner. Deworming drugs for soil-transmitted intestinal worms in children: effects on nutritional indicators, haemoglobin, and school performance. Cochrane Database of Systematic Reviews, (7), 2015.

[10] Kevin Croke, Joan Hamory Hicks, Eric Hsu, Michael Kremer, and Edward Miguel. Does mass deworming affect child nutrition? Meta-analysis, cost-effectiveness, and statistical power. The World Bank, 2016. 
[11] Muhammad Farhan Majid, Su Jin Kang, and Peter J Hotez. Resolving "worm wars": An extended comparison review of findings from key economics and epidemiological studies. PLoS Neglected Tropical Diseases, 13(3):e0006940, 2019.

[12] Joachim Hartung and Guido Knapp. On tests of the overall treatment effect in metaanalysis with normally distributed responses. Statistics in Medicine, 20(12):1771-1782, 2001.

[13] Joanna IntHout, John PA Ioannidis, and George F Borm. The Hartung-Knapp-SidikJonkman method for random effects meta-analysis is straightforward and considerably outperforms the standard DerSimonian-Laird method. BMC Medical Research Methodology, 14(1):1, 2014.

[14] Rebecca J Hardy and Simon G Thompson. Detecting and describing heterogeneity in meta-analysis. Statistics in Medicine, 17(8):841-856, 1998.

[15] Samuel S Shapiro and RS Francia. An approximate analysis of variance test for normality. Journal of the American Statistical Association, 67(337):215-216, 1972. 